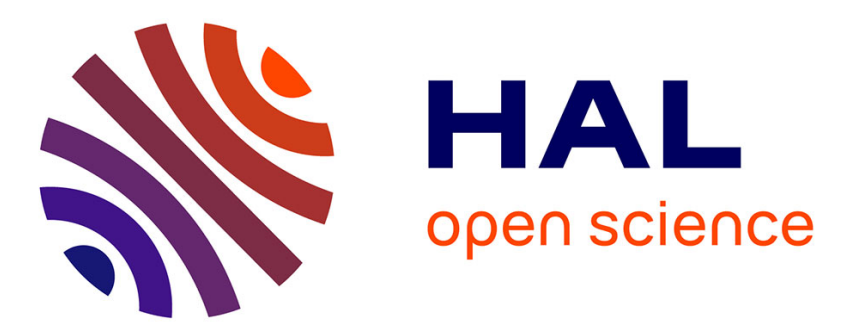

\title{
Bounds and correlation approximation for the effective conductivity of heterogeneous plates
}

\author{
H. Le Quang, Guy Bonnet, D. C. Pham
}

\section{To cite this version:}

H. Le Quang, Guy Bonnet, D. C. Pham. Bounds and correlation approximation for the effective conductivity of heterogeneous plates. Physical Review E: Statistical, Nonlinear, and Soft Matter Physics, 2011, 84 (6), pp.06153. 10.1103/PhysRevE.84.061153 . hal-00692876

\section{HAL Id: hal-00692876 https://hal.science/hal-00692876}

Submitted on 6 Mar 2016

HAL is a multi-disciplinary open access archive for the deposit and dissemination of scientific research documents, whether they are published or not. The documents may come from teaching and research institutions in France or abroad, or from public or private research centers.
L'archive ouverte pluridisciplinaire HAL, est destinée au dépôt et à la diffusion de documents scientifiques de niveau recherche, publiés ou non, émanant des établissements d'enseignement et de recherche français ou étrangers, des laboratoires publics ou privés. 


\title{
Bounds and correlation approximation for the effective conductivity of heterogeneous plates
}

\author{
H. Le Quang and G. Bonnet* \\ Universite Paris-Est, Laboratoire Modelisation et Simulation Muiti Echelle, MSME UMR 8208 CNRS, 5 Boulevard Descartes, \\ F-77454 Marne-la-Vallee Cedex 2, France \\ D. C. Pham \\ Institute of Mechanics-VAST, 264 Doi Can, Hanol, Vietnam
}

The size effect obtained when studying the effective properties of plates is investigated by producing a third-order correlation approximation and Hashin-Shtrikman bounds for the effective in-plane conductivity of heterogeneous plates. The boundary condition of the plates is taken into account by obtaining the exact Green operator for the boundary problem. Results are obtained for a two-dimensional (2D) random distribution of disks and a 3D distribution of spheres. All results recover those obtained for an infinite medium when the heterogeneity size becomes small compared to plate thickness. They show that the size effect is more significant in the case of a $2 \mathrm{D}$ distribution of heterogeneities than for a $3 \mathrm{D}$ distribution.

PACS number(s): 46.65.+g, 72.80.Tm, 72.80.Ng, 71.15.Mb

\section{INTRODUCTION}

Numerous methods can be used to predict the effective properties of heterogeneous materials. In the case of materials with randomly distributed properties in large domains, bounds of the effective properties, which take into account secondor third-order correlation functions, have been provided in [1-7]. Alternatively, different kinds of approximations can be used, including Maxwell, self-consistent, differential scheme [8-11], and those involving correlation information about the microgeometries of the composites [2,3,7,12-16]. The perturbation series in the component properties' contrast is often used when the contrast is small $[2,3]$, while for highcontrast-property composites, the strong-contrast expansion is developed [7,17], which is expected to have a larger radius of convergence than the weak-contrast one. It is interesting to note that a series expansion on the contrast parameter converges for any nonoverlapping distribution of the disks on the plane [14]. Still, in practice we have to restrict ourselves to approximations involving up to three-point correlation parameters, since higher-point correlation information is not available for most practical microstructures.

Sen and Torquato [18] derived strong-contrast expansions for the effective conductivity tensor of macroscopically anisotropic two-phase media. Pham and Torquato [19] extended this approach further to the $N$-phase composites. From the expansions, they proposed the three-point correlation approximation for the effective conductivity of isotropic composites that, in the case of two-phase materials, agrees well with a number of analytical and numerical results, even when the contrast between the phases is infinite and their volume proportions are near percolation thresholds. The simple approximation reduces to the well-known Maxwell and self-consistent ones for the respective asymmetric matrix mixture and symmetric cell microgeometries, and it obeys Hashin-Shtrikman as well as three-point correlation bounds over all the parameter ranges.

¿guy.bonnet@univ-paris-est.fr
It is important to evaluate the effect of restricted domains such as plates when the size of the heterogeneities is no longer negligible compared to a characteristic size of the domain (e.g., the thickness of the plate). In the case of plates, first- and second-order bounds were extended in the case of elastic properties [20-23]. The aim of this paper is to provide additional results on the conductivity of plates, taking into account simultaneously up to third-order correlations and the effect of plate thickness.

In Sec. II, a third-order correlation expansion in the case of large domains is displayed, which uses a strong-contrast expansion of the effective properties. The subsequent sections are devoted to studying the conductivity of heterogeneous plates. Taking into account boundary conditions at the surface of the plate can be done effectively by using a Green operator, as was done in [23]. This Green operator for homogeneous plates is computed in Sec. III. It is then possible to obtain second-order Hashin-Shtrikman bounds for the conductivity of plates (Sec. IV) and an approximation involving secondand third-order correlations (Sec. V) based on the approach described in Sec. II. Numerical applications are provided in Sec. VI, allowing us to evaluate the effect of plate thickness on in-plane conductivity. A few concluding comments are presented in Sec. VII.

\section{STRONG-CONTRAST EXPANSION AND CORRELATION APPROXIMATION}

For the reasons mentioned in the Introduction, our correlation approximation will be based on the strong-contrast expansion.

Following [7,19], let us consider a macroscopically anisotropic two-phase composite in space dimension $d(d=$ 2 or 3) comprised of isotropic phases having conductivities $c_{\alpha}$ and volume fractions $v_{\alpha}(\alpha=1$ or 2$)$. The local scalar conductivity at position $\mathbf{x}$ is expressible as

$$
c(\mathbf{x})=\sum_{\alpha=1}^{2} c_{\alpha} \mathcal{I}^{(\alpha)}(\mathbf{x}),
$$


where

$$
\mathcal{I}^{(\alpha)}(\mathbf{x})= \begin{cases}1 & \text { if } \mathbf{x} \text { is in phase } \alpha, \\ 0 & \text { otherwise, }\end{cases}
$$

is the indicator function for phase $\alpha$ ( $\alpha=1$ or 2). For statistically homogeneous media, $\left\langle\mathcal{I}^{(\alpha)}(\mathbf{x})\right\rangle=v_{\alpha}$, where angular brackets denote an ensemble average. The local conductivity $c(\mathbf{x})$ is the coefficient of proportionality in the linear constitutive relation

$$
\mathbf{q}(\mathbf{x})=c(\mathbf{x}) \mathbf{E}(\mathbf{x})
$$

between the local thermal flux $\mathbf{q}$ at position $\mathbf{x}$ and the local field intensity $\mathbf{E}(\mathbf{x})$, which is the opposite of the gradient of temperature field $T(\mathbf{x})$,

$$
\mathbf{E}(\mathbf{x})=-\nabla T(\mathbf{x}),
$$

while the flux should satisfy the energy conservation equation

$$
\nabla \cdot q(x)=0
$$

in the case of stationary thermal conduction without a heat source.

Let us consider this $d$-dimensional composite specimen with an infinite reference medium having conductivity $c_{0}$, which is subjected to an applied intensity field $\mathbf{E}^{0}$ at infinity, and introduce the Green operator for the reference medium

$$
\mathbf{G}(\mathbf{r})=\mathbf{D} \delta(\mathbf{r})-\mathbf{H}(\mathbf{r}) \text {, }
$$

where $\mathbf{D}=\mathbf{I} / d c_{0}, \mathbf{r}=\mathbf{x}-\mathbf{x}^{\prime}, \mathbf{n}=\mathbf{r} /|\mathbf{r}|, \delta(\mathbf{r})$ is the delta Dirac function, and $\mathbf{I}$ is the second-order identity tensor. The constant second-order tensor $\mathbf{D}$ arises because of the exclusion of the spherical cavity, and it is understood that integrals involving the second-order traceless tensor $\mathbf{H}$ are to be carried out by excluding at $\mathbf{x}^{\prime}=\mathbf{x}$ an infinitesimal sphere in the limit in which the sphere radius shrinks to zero (Cauchy principal part). For the infinite space, the second-order tensor $\mathbf{H}$ can be expressed explicitly by

$$
\mathbf{H}(\mathbf{r})=\frac{1}{2(d-1) \pi c_{0}} \frac{d \mathbf{n} \otimes \mathbf{n}-\mathbf{I}}{r^{d}} .
$$

Moreover, the integral of $\mathbf{H}(\mathbf{r})$ over the surface of a sphere of radius $R>0$ is identically zero, i.e., $\int_{r=R} \mathbf{H}(\mathbf{r}) d \Omega=0$.

For later use, it is convenient to denote

$$
\begin{gathered}
L(\mathbf{x})=c_{0} d \frac{c(\mathbf{x})-c_{0}}{c(\mathbf{x})+(d-1) c_{0}}=c_{0} d \sum_{\alpha=1}^{2} b_{\alpha 0} \mathcal{I}^{(\alpha)}(\mathbf{x}), \\
b_{\alpha 0}=\frac{c_{\alpha}-c_{0}}{c_{\alpha}+(d-1) c_{0}}, \\
\mathbf{L}^{e}=c_{0} d\left[\mathbf{C}^{e}-c_{0} \mathbf{I}\right] \cdot\left[\mathbf{C}^{e}+(d-1) c_{0} \mathbf{I}\right]^{-1},
\end{gathered}
$$

where $\mathbf{C}^{e}$ is the effective conductivity tensor of the macroscopically anisotropic composite.

Following the approach presented in $[7,19]$, we obtain the result for the effective tensor $\mathbf{L}^{c}$,

$$
\left(\mathbf{L}^{c}\right)^{-1}=\mathbf{H}+\left\langle L(\mathbf{I}-L \mathbf{H})^{-1}\right\rangle^{-1},
$$

or in the infinite series expansion, the first few terms of which are explicitly given by (we adopt the shorthand notation of representing $\mathbf{x}$ and $\mathbf{x}^{\prime}$ by 1 and $2, \ldots$, respectively)

$$
\begin{aligned}
\left(\mathbf{L}^{\mathcal{C}}\right)^{-1}(1)= & \frac{\mathbf{I}(1)}{\langle L(1)\rangle}-\int d 2 \frac{\langle L(1) L(2)\rangle-\langle L(1)\rangle\langle L(2)\rangle}{\langle L(1)\rangle\langle L(2)\rangle} \mathbf{H}(1,2)-\iint d 2 d 3\left(\frac{\langle L(1) L(2) L(3)\rangle}{\langle L(1)\rangle\langle L(2)\rangle}-\frac{\langle L(1) L(2)\rangle\langle L(2) L(3)\rangle}{\langle L(1)\rangle\langle L(2)\rangle\langle L(3)\rangle}\right) \\
& \times \mathbf{H}(1,2) \cdot \mathbf{H}(2,3)-\cdots .
\end{aligned}
$$

The general term contains the $n$-point correlation functions $\langle L(1) \cdots L(n)\rangle$. Multiplying Eq. (12) by $c_{0}$, truncating the series after the third-order term, and making the substitution into Eq. (10), we get the third-order approximation equation

$$
\left(\frac{1}{c_{0}} \mathbf{C}^{e}-\mathbf{I}\right)^{-1}+\frac{1}{d} \mathbf{I}=\frac{1}{d B} \mathbf{I}-\mathbf{A}_{2}-\mathbf{A}_{3},
$$

where (assuming statistical homogeneity)

$$
B=\sum_{\alpha=1}^{2} v_{\alpha} b_{\alpha 0},
$$

$\mathbf{A}_{2}$ and $\mathbf{A}_{3}$ are, respectively, second- and third-order terms (the higher-order terms have been neglected),

$$
\begin{aligned}
\mathbf{A}_{2}= & \frac{1}{B^{2}} \int d 2 \sum_{\alpha, \beta=1}^{2} b_{\alpha 0} b_{\beta 0}\left[\left(\mathcal{I}^{(\alpha)}(1) \mathcal{I}^{(\beta)}(2)\right\rangle\right. \\
& \left.-\left\langle\mathcal{I}^{(\alpha)}(1)\right\rangle\left\langle\mathcal{I}^{(\beta)}(2)\right\rangle\right] \mathbf{h}(1,2) \\
= & \frac{\left(b_{20}-b_{10}\right)^{2}}{B^{2}} \int d 2\left[S_{2}^{(22)}(1,2)-v_{2}^{2}\right] \mathbf{h}(1,2),
\end{aligned}
$$

with $S_{2}^{(\alpha \beta)}(1,2)=\left\langle\mathcal{I}^{(\alpha)}(1) \mathcal{I}^{(\beta)}(2)\right\rangle$ specifying the respective two-point correlation function, and

$$
\begin{aligned}
& \mathbf{A}_{3}= \frac{1}{B^{2}} \iint d 2 d 3\left[\sum_{\alpha, \beta, \gamma=1}^{2} b_{\alpha 0} b_{\beta 0} b_{\gamma 0}\left\langle\mathcal{I}^{(\alpha)}(1) \mathcal{I}^{(\beta)}(2) \mathcal{I}^{(\gamma)}(3)\right\rangle\right. \\
&-\frac{1}{B} \sum_{\alpha, \beta, \gamma, \delta=1}^{2} b_{\alpha 0} b_{\beta 0} b_{\gamma 0} b_{\delta 0}\left\langle\mathcal{I}^{(\alpha)}(1) \mathcal{I}^{(\beta)}(2)\right\rangle \\
&\left.\times\left\langle\mathcal{I}^{(\gamma)}(2) \mathcal{I}^{(\delta)}(3)\right\rangle\right] \mathbf{h}(1,2) \mathbf{h}(2,3), \\
& \mathbf{h}(\mathbf{r})=c_{0} \mathbf{H}(\mathbf{r}) .
\end{aligned}
$$

For macroscopically isotropic infinite media, $\mathbf{A}_{2}=\mathbf{0}$ since $\mathbf{h}$, like $\mathbf{H}$, is traceless. We take the trace of both sides of Eq. (13) and choose the conductivity $c_{0}$ of the reference medium as the solution of the equation

$$
\sum_{\alpha=1}^{2} \xi_{\alpha} \frac{c_{\alpha}-c_{0}}{c_{\alpha}+(d-1) c_{0}}=0,
$$


which makes the trace of $\mathbf{A}_{3}$ vanish. Then we get the threepoint correlation approximation for the effective conductivity $c_{e}$ of the macroscopically isotropic media [19],

$$
c_{e}=\left[\sum_{\alpha=1}^{2} \frac{v_{\alpha}}{c_{\alpha}+(d-1) c_{0}}\right]^{-1}-(d-1) c_{0} ;
$$

alternative expressions of the geometric three-point correlation parameters $\xi_{1}, \xi_{2}\left(\xi_{1}, \xi_{2} \geqslant 0, \xi_{1}+\xi_{2}=1\right)$ in Eq. (18) and tables of their values for various microgeometries are given in $[7,19]$. For example, a very good approximation of $\xi_{2}$ versus particle volume fraction $v_{2}$ can be given as

$$
\xi_{2}=0.21068 v_{2}-0.04693 v_{2}^{2}+0.00247 v_{2}^{3},
$$

with $v_{2} \leqslant 0.6$ for the random suspension of equisized hard spheres in a matrix or provided by

$$
\xi_{2}=\frac{1}{3} v_{2}-0.05707 v_{2}^{2},
$$

with $v_{2} \leqslant 0.7$ for the random distribution of unidirectionally aligned, infinitely long identical circular hard fibers in a matrix.

Approximation (19), with $c_{0}$ determined by Eq. (18), involves the volume fractions $v_{1}, v_{2}$ and the conductivities $c_{1}, c_{2}$ of the two components as well as the three-point correlation parameters $\xi_{1}, \xi_{2}$ describing the microgeometry of the isotropic two-component composite. The three-point correlation approximation falls within both the second-order Hashin-Shtrikman bounds and third-order bounds involving three-point correlation parameters $\xi_{1}, \xi_{2}$ over all the parameter ranges [19].

This simple approximation reduces to the well-known self-consistent one for the respective symmetric cell microgeometries $\left(\xi_{2}=v_{2}\right)$, as well as to the Maxwell approximation for the asymmetric matrix mixtures $\left(\xi_{2}=0\right)$ that coincides with the Hashin-Shtrikman upper (or lower) bound when the matrix phase (phase 1) is the phase of larger (or smaller) conductivity.

The approximation agrees well with simulation data for periodic structures as well as random suspensions of equalized hard or overlapping random spheres for the highest contrast

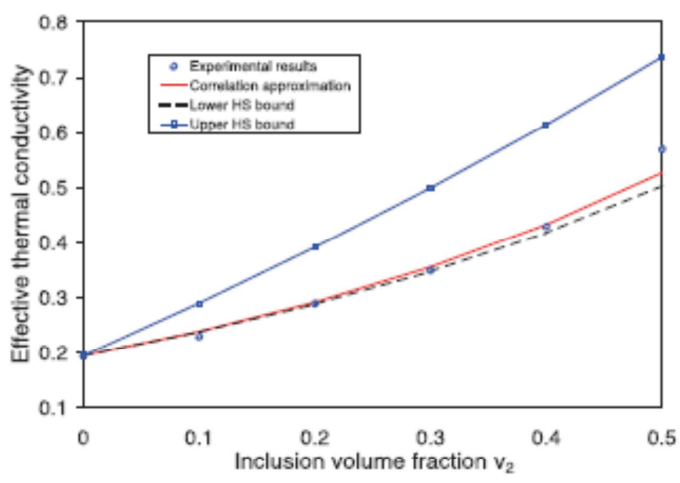

FIG. 1. (Color online) The thermal conductivity obtained by the three-point correlation approximation is compared with the experimental results and Hashin-Shtrikman bounds for a random suspension of silica spherical particles (conductivity $1.5 \mathrm{~W} / \mathrm{mK}$ ) in a polymer matrix (conductivity $0.195 \mathrm{~W} / \mathrm{mK}$ ). composites involving superconductive or insulate phases, up to the high volume fractions of the inclusion phase near percolation thresholds (see, e.g., [19]).

Here, as an additional illustration, we compare in Fig. 1 the predictions of the three-point correlation approximation (19) accounting for (18) and (20) with the experimental results by [24] for the high contrast composites made of a polymer matrix (thermal conductivity $0.195 \mathrm{~W} / \mathrm{mK}$ ) reinforced with silica spherical particles (conductivity $1.5 \mathrm{~W} / \mathrm{mK}$ ). HashinShtrikman bounds are also included for comparison.

A modification of the procedure for the plates of finite thickness will be developed thereafter in Sec. V.

\section{A GREEN OPERATOR FOR ISOLATED PLATES AND PERIODIC POLARIZATIONS}

To compute Hashin-Shtrikman-type bounds (Sec. IV) as well as the correlation approximation (Sec. V) for the conductivity of composite plates with a possible finite-size (thickness) effect, we need to construct the Green operator for the plates.

The Green operator for the plates obtained in this section can be considered to be an extension of the thermal conduction phenomenon of the result of [25] for the context of linear elasticity. Moreover, the method elaborated and results obtained by our work for the Green operator of the plates can be used in the thermal conduction phenomenon, but they are also applicable to physically analogous transport phenomena, such as electric conduction, dielectrics, magnetism, diffusion, and flow in porous media.

The Green operator for the plate can be obtained as the limit for large periods of the Green operator for periodic cells, which can be used, for example, for obtaining the properties of heterogeneous periodic plates. Indeed, we consider now a homogeneous plate of conductivity $c_{0}$ that is assumed to be subjected to a polarization field that is periodic in the $x_{1}-x_{2}$ plane of the plate. This plate can, therefore, be characterized by a unit cell denoted by $Z$ and defined by

$$
\begin{array}{r}
Z=\left\{\mathbf{x} \in \mathbf{R}^{3}, \mathbf{x}=\left(x_{1}, x_{2}, x_{3}\right), x_{\alpha} \in\right. \\
\quad]-\frac{l_{\alpha}}{2}, \frac{l_{\alpha}}{2}\left[, x_{3} \in\right]-\frac{t}{2}, \frac{t}{2}[\},
\end{array}
$$

where $\alpha=1$ or $2 ; l_{1}, l_{2}$, and $t$ are the length, width, and thickness of $Z$, respectively. We denote by $\omega=]-l_{1} / 2, l_{1} / 2[\times$ ] $-l_{2} / 2, l_{2} / 2$ [ the middle surface of $Z$ and by $\partial \omega$ the boundary of $\omega$. The lateral boundary $\partial Z_{l}$ of $Z$ is defined by $\partial Z_{l}=$ $\partial \omega \times]-t / 2, t / 2\left[\right.$. The top and bottom surfaces $\partial Z^{ \pm}$of $Z$ are defined by $\partial Z^{ \pm}=\omega \times( \pm t / 2)$. A mixed boundary condition with a periodic boundary condition on $\partial Z_{l}$ and an insulation boundary condition on $\partial Z^{ \pm}$is imposed on the external surface of $Z$. Simultaneously, a periodic polarization field $\mathbf{p}(\mathbf{x})$ is applied. For this problem, the intensity solution field $\mathbf{E}(\mathbf{x})$ is related linearly to the periodic polarization field $\mathbf{p}(\mathbf{x})$ as follows:

$$
\mathbf{E}(\mathbf{x})=-\int_{Z} \mathbf{G}\left(\mathbf{x}-\mathbf{x}^{\prime}\right) \mathbf{p}\left(\mathbf{x}^{\prime}\right) d \mathbf{x}^{\prime},
$$

where $\mathbf{G}$ is the Green operator with insulation boundary conditions. Moreover, this intensity solution field $\mathbf{E}(\mathbf{x})$ can be 
decomposed into two parts, i.e., $\mathbf{E}(\mathbf{x})=\mathbf{E}^{p}(\mathbf{x})+\mathbf{E}^{c}(\mathbf{x})$. The first part, $\mathbf{E}^{p}(\mathbf{x})$, corresponds to the intensity solution field of the periodic boundary value problem in which the unit cell $Z$ medium with periodic boundary conditions on $\partial Z_{l}$ and $\partial Z^{ \pm}$is imposed on the periodic polarization field $\mathbf{p}(\mathbf{x})$. This problem can be expressed in the form

$$
\begin{aligned}
& \nabla \cdot \mathbf{q}^{p}(\mathbf{x})=\mathbf{0} \quad \text { in } \mathbf{Z}, \\
& \mathbf{q}^{p}(\mathbf{x})=c_{0} \mathbf{E}^{p}(\mathbf{x})+\mathbf{p}(\mathbf{x}) \quad \text { in } Z, \\
& \mathbf{E}^{p}(\mathbf{x})=-\nabla T^{p}(\mathbf{x}) \quad \text { in } Z, \\
& T^{p}(\mathbf{x}) \text { periodic on } \partial Z_{l}, \\
& \mathbf{q}^{p}(\mathbf{x}) \cdot \mathbf{n} \text { antiperiodic on } \partial Z_{l}, \text { and } \partial Z^{ \pm} .
\end{aligned}
$$

By introducing the Green operator $\mathbf{G}^{P}$ with periodic boundary conditions, the intensity solution field $\mathbf{E}^{p}(\mathbf{x})$ takes the following form:

$$
\mathbf{E}^{p}(\mathbf{x})=-\int_{Z} \mathbf{G}^{p}\left(\mathbf{x}-\mathbf{x}^{\prime}\right) \mathbf{p}\left(\mathbf{x}^{\prime}\right) d \mathbf{x}^{\prime} .
$$

It is clear that when the dimensions of the unit cell $Z$ are large enough, the Green operator $\mathbf{G}^{p}$ for the problem (24) becomes equal to the Green operator for an infinite medium provided by Eqs. (6) and (7).

The second part, $\mathbf{E}^{c}(\mathbf{x})$, corresponds to the intensity solution field of the complementary boundary value problem, which is necessary to comply with the insulation boundary conditions on $\partial Z^{ \pm}$. This complementary problem is defined as

$$
\begin{aligned}
& \nabla \cdot \mathbf{q}^{c}(\mathbf{x})=\mathbf{0} \quad \text { in } \mathbf{Z}, \\
& \mathbf{q}^{c}(\mathbf{x})=c_{0} \mathbf{E}^{c}(\mathbf{x}) \quad \text { in } Z, \\
& \mathbf{E}^{c}(\mathbf{x})=-\nabla T^{c}(\mathbf{x}) \quad \text { in } Z, \\
& T^{c}(\mathbf{x}) \text { periodic on } \partial Z_{l}, \\
& \mathbf{q}^{c}(\mathbf{x}) \cdot \mathbf{n} \text { antiperiodic on } \partial Z_{l}, \\
& q_{3}^{c}\left(x_{1}, x_{2}, \pm \frac{t}{2}\right)=-q_{3}^{p}\left(x_{1}, x_{2}, \pm \frac{t}{2}\right),
\end{aligned}
$$

where $q_{3}^{p}\left(x_{1}, x_{2}, \pm \frac{t}{2}\right)$ is the third component of the heat-flux solution field of (24). The intensity solution field $\mathbf{E}^{c}(\mathbf{x})$ of (26) can be written in the following form:

$$
\mathbf{E}^{c}(\mathbf{x})=-\int_{Z} \mathbf{G}^{c}\left(\tilde{\mathbf{x}}-\tilde{\mathbf{x}}^{\prime}, x_{3}, x_{3}^{\prime}\right) \mathbf{p}\left(\mathbf{x}^{\prime}\right) d \mathbf{x}^{\prime},
$$

where $\tilde{\mathbf{x}}=\left(x_{1}, x_{2}\right)$ and $\tilde{\mathbf{x}}^{\prime}=\left(x_{1}^{\prime}, x_{2}^{\prime}\right)$; the Green operator $\mathbf{G}^{c}\left(\tilde{\mathbf{x}}-\tilde{\mathbf{x}}^{\prime}, x_{3}, x_{3}^{\prime}\right)$ is periodic in the $x_{1}-x_{2}$ plane. Thus, the Green operator $\mathbf{G}$ with free heat flux boundary conditions is given in the form

$$
\mathbf{G}=\mathbf{G}^{P}+\mathbf{G}^{c} \text {. }
$$

For later use, we note from the problem (24) that $\mathbf{G}$ and $\mathbf{G}^{p}$ are two self-adjoint operators. Consequently, the Green operator $\mathbf{G}^{c}$ related to $\mathbf{G}$ and $\mathbf{G}^{p}$ by Eq. (28) is also a self-adjoint operator. Moreover, the explicit expressions of $\mathbf{G}^{c}$ and $\mathbf{G}^{p}$ have been derived in the Appendix.

\section{HASHIN-SHTRIKMAN-TYPE BOUNDS FOR THE PLATE'S IN-PLANE CONDUCTIVITY}

In this section, the two materials composing the heterogeneous plate under consideration are assumed to be randomly distributed. Additionally, on a macroscopic scale, this heterogeneous plate is supposed to be statically homogeneous along directions in the $x_{1}-x_{2}$ plane. By taking into account two-point correlation information about the microstructure and boundary conditions of the plate and by applying the Hashin-Shtrikman variational principle, we aim at determining the upper and lower bounds for the effective conductivity of this random plate.

\section{A. Hashin-Shtrikman variational principle}

We start by introducing the thermal energy $W_{\text {eff }}$, which is defined as

$$
W_{\text {eff }}=\frac{1}{2|Y|} \int_{Y} \mathbf{q}(\mathbf{x}) \cdot \mathbf{E}(\mathbf{x}) d \mathbf{x}=\frac{1}{2} \mathbf{E}^{0} \cdot \mathbf{C}^{\epsilon} \mathbf{E}^{0},
$$

where $Y$ is a representative volume element (RVE) of the heterogeneous plate under consideration, $\mathbf{E}^{0}$ is the applied uniform intensity field on the boundary of the plate, and $\mathbf{q}(\mathbf{x})$ and $\mathbf{E}(\mathbf{x})$ are, respectively, the flux and intensity solution fields.

Next, we define a trial function $W(\boldsymbol{\tau})$ in the following form:

$$
W(\boldsymbol{\tau})=W_{0}+W_{1}(\boldsymbol{\tau})
$$

where

$$
\begin{gathered}
W_{0}=\frac{1}{2} c_{0} \mathbf{E}^{0} \cdot \mathbf{E}^{0}, \\
W_{1}(\boldsymbol{\tau})=\frac{1}{2|Y|} \int_{Y}\left(2 \boldsymbol{\tau} \cdot \mathbf{E}^{0}-\delta c^{-1} \boldsymbol{\tau} \cdot \boldsymbol{\tau}-\boldsymbol{\tau} \cdot \mathbf{G} \boldsymbol{\tau}\right) d \mathbf{x},
\end{gathered}
$$

with $\delta c=c(\mathbf{x})-c_{0}$, and $\boldsymbol{\tau}(\mathbf{x})$ being a trial periodic polarization field. It is noted that the function $W_{0}$ is independent of the trial periodic polarization field $\boldsymbol{\tau}(\mathbf{x})$, and that the trial function $W(\tau)$ will be equal to $W_{\text {eff }}$ if and only if the trial periodic polarization field is equal to the polarization corresponding to the solution field, i.e., $\boldsymbol{\tau}(\mathbf{x})=\mathbf{p}(\mathbf{x})=\delta c \mathbf{E}^{0}$.

By applying the well-known Hashin-Shtrikman variational principle [1], it can be shown that

$$
W_{\text {eff }}=\frac{1}{2} \mathbf{E}^{0} \cdot \mathbf{C}^{\boldsymbol{c}} \mathbf{E}^{0}= \begin{cases}\max _{\boldsymbol{\tau}}\left\{W_{0}+W_{1}(\boldsymbol{\tau})\right\}, & \delta c \geqslant 0, \\ \min _{\boldsymbol{\tau}}\left\{W_{0}+W_{1}(\boldsymbol{\tau})\right\}, & \delta c \leqslant 0 .\end{cases}
$$

Finally, since the function $W_{0}$ is independent of $\tau(\mathbf{x})$, the upper and lower bounds of the effective conductivity tensor $\mathbf{C}^{e}$ for the random plate are then obtained by finding the trial periodic polarization field $\boldsymbol{\tau}(\mathbf{x})$ in the same way the function $W_{1}(\tau)$ attains its maximum value in the case in which $\delta c \geqslant 0$ and its minimum value in the case in which $\delta c \leqslant 0$, respectively.

\section{B. Choice of the trial polarization field}

As proven in Sec. IV A, an important feature necessary to obtain the upper and lower Hashin-Shtrikman bounds is the choice of the trial periodic polarization field $\boldsymbol{\tau}(\mathbf{x})$. We will show in this subsection that the trial periodic polarization field $\boldsymbol{\tau}(\mathbf{x})$ can be found by estimating the stationary value of $W_{1}(\tau)$ as below. 
First, the trial periodic polarization field in each phase is chosen so that it is independent of $x_{1}$ and $x_{2}$, i.e., $\boldsymbol{\tau}_{\alpha}(\mathbf{x})=$ $\boldsymbol{\tau}_{\alpha}\left(x_{3}\right)$ with $\alpha=1$ or 2 . Moreover, owing to the fact that the random plate is statistically homogeneous along directions in the $x_{1}-x_{2}$ plane, the characteristic properties are invariant with respect to any translation along these directions, i.e.,

$$
\begin{gathered}
\left\langle\mathcal{I}^{(\alpha)}(\mathbf{x})\right\rangle=\left\langle\mathcal{I}^{(\alpha)}\left(x_{3}\right)\right\rangle=S_{1}^{(\alpha)}\left(x_{3}\right) \\
\left\langle\mathcal{I}^{(\alpha)}(\mathbf{x}) \mathcal{I}^{(\beta)}\left(\mathbf{x}^{\prime}\right)\right\rangle=S_{2}^{(\alpha \beta)}\left(\mathbf{x}, \mathbf{x}^{\prime}\right)=S_{2}^{(\alpha \beta)}\left(\tilde{\mathbf{x}}-\tilde{\mathbf{x}}^{\prime}, x_{3}, x_{3}^{\prime}\right)
\end{gathered}
$$

An ensemble average of Eq. (31), taking into account Eqs. (28), (34), and (34), yields

$$
\begin{aligned}
\left\langle W_{1}(\tau)\right\rangle & \frac{1}{2|Y|} \int_{Y}\left[2 \sum_{\alpha=1}^{2} S_{1}^{(\alpha)}\left(x_{3}\right) \boldsymbol{\tau}_{\alpha}\left(x_{3}\right) \cdot \mathbf{E}^{0}\right. \\
& -\sum_{\alpha=1}^{2} S_{1}^{(\alpha)}\left(x_{3}\right) \delta c^{-1} \boldsymbol{\tau}_{\alpha}\left(x_{3}\right) \cdot \boldsymbol{\tau}_{\alpha}\left(x_{3}\right) \\
& -\sum_{\alpha, \beta=1}^{2} \boldsymbol{\tau}_{\alpha}\left(x_{3}\right) \int_{Y} \mathbf{G}^{P}\left(\tilde{\mathbf{x}}-\tilde{\mathbf{x}}^{\prime}, x_{3}, x_{3}^{\prime}\right) \boldsymbol{\tau}_{\beta}\left(x_{3}^{\prime}\right) S_{2}^{(\alpha \beta)} d \mathbf{x}^{\prime} \\
& \left.-\sum_{\alpha, \beta=1}^{2} \boldsymbol{\tau}_{\alpha}\left(x_{3}\right) \int_{Y} \mathbf{G}^{c}\left(\tilde{\mathbf{x}}-\tilde{\mathbf{x}}^{\prime}, x_{3}, x_{3}^{\prime}\right) \boldsymbol{\tau}_{\beta}\left(x_{3}^{\prime}\right) S_{2}^{(\alpha \beta)} d \mathbf{x}^{\prime}\right] d \mathbf{x} .
\end{aligned}
$$

Second, we discretize the plate under consideration along the $x_{3}$ and $x_{3}^{\prime}$ directions with $N_{3}$ points each. Correspondingly, the trial periodic polarization field in each phase is approximately expressed by a piecewise constant function such as

$$
\tau_{\alpha}\left(x_{3}\right)=\sum_{m=1}^{N_{3}} \tau_{\alpha}^{m} \mathcal{I}_{m}\left(x_{3}\right),
$$

where $\mathcal{I}_{m}\left(x_{3}\right)$ is an indicator function of the finite intervals whose union yields the part of the $x_{3}$ axis that is inside the plate. Thus, Eq. (36) is then rewritten as

$$
\begin{aligned}
& \left\langle W_{1}(\boldsymbol{\tau})\right\rangle \\
& =\frac{1}{2 N_{3}} \sum_{m=1}^{N_{3}}\left[\sum_{\alpha=1}^{2}\left(2 S_{\alpha}^{m} \boldsymbol{\tau}_{\alpha}^{m} \cdot \mathbf{E}^{0}-S_{\alpha}^{m} \delta c^{-1} \boldsymbol{\tau}_{\alpha}^{m} \cdot \boldsymbol{\tau}_{\alpha}^{m}\right)-\sum_{m^{\prime}=1}^{N_{3}}\right. \\
& \left.\quad \times \sum_{\alpha, \beta=1}^{2} \frac{\boldsymbol{\tau}_{\alpha}^{m} \boldsymbol{\tau}_{\beta}^{m \prime^{\prime}}}{N_{3}} \int_{\omega}\left[\mathbf{G}_{p}^{m m^{\prime}}\left(\tilde{\mathbf{x}}^{\prime}\right)+\mathbf{G}_{c}^{m m^{\prime}}\left(\tilde{\mathbf{x}}^{\prime}\right)\right] S_{\alpha \beta}^{m m m^{\prime}}\left(\tilde{\mathbf{x}}^{\prime}\right) d \tilde{\mathbf{x}}^{\prime}\right],
\end{aligned}
$$

where $\quad \mathbf{G}_{p}^{m m^{\prime}}\left(\tilde{\mathbf{x}}^{\prime}\right)=\mathbf{G}^{p}\left(\tilde{\mathbf{x}}^{\prime}, x_{3}(m)-x_{3}^{\prime}\left(m^{\prime}\right)\right), \quad \mathbf{G}_{c}^{m m^{\prime}}\left(\tilde{\mathbf{x}}^{\prime}\right)=$ $\mathbf{G}^{c}\left(\tilde{\mathbf{x}}^{\prime}, x_{3}(m), x_{3}^{\prime}\left(m^{\prime}\right)\right), S_{\alpha \beta}^{m m^{\prime}}\left(\tilde{\mathbf{x}}^{\prime}\right)=S_{2}^{(\alpha \beta)}\left(\tilde{\mathbf{x}}^{\prime}, x_{3}(m), x_{3}^{\prime}\left(m^{\prime}\right)\right)$, and $S_{\alpha}^{m}=S_{1}^{(\alpha)}\left[x_{3}(m)\right]$, with $x_{3}(m)$ and $x_{3}^{\prime}\left(m^{\prime}\right)$ standing for the third coordinates of the $m$ th and $m^{\prime}$ th discretized points, respectively.

Third, the surface integral in Eq. (38) can be calculated by using the fast Fourier transform (FFT) with the wave vectors $\mathbf{k}=\left(k_{1}, k_{2}\right)$ and by applying the Parseval theorem.
The expression of Eq. (38) is finally rewritten in the simple form

$$
\begin{aligned}
& \left\langle W_{1}(\boldsymbol{\tau})\right\rangle \\
& =\frac{1}{2 N_{3}}\left(2 \mathbf{T}^{T} \cdot \mathbf{F}-\mathbf{T}^{T} \cdot \mathbf{M}_{v} \mathbf{T}-\mathbf{T}^{T} \cdot \mathbf{M}_{p} \mathbf{T}-\mathbf{T}^{T} \cdot \mathbf{M}_{c} \mathbf{T}\right) .
\end{aligned}
$$

where the discretized trial polarization vector $\mathbf{T}$ is defined by

$$
\mathbf{T}=\left(\boldsymbol{\tau}_{1}^{1}, \boldsymbol{\tau}_{2}^{1}, \boldsymbol{\tau}_{1}^{2}, \tau_{2}^{2}, \ldots, \boldsymbol{\tau}_{1}^{N_{3}}, \boldsymbol{\tau}_{2}^{N_{3}}\right)
$$

with $\boldsymbol{\tau}_{\alpha}^{m}=\boldsymbol{\tau}_{\alpha}\left[x_{3}(m)\right]$; the vector $\mathbf{F}$ is determined from the one-point correlation function $S_{\alpha}^{m}$ and the applied uniform intensity field $\mathbf{E}^{0}, \mathbf{M}_{v}$ is a symmetric squared matrix to be calculated from the one-point correlation functions $S_{1}^{(\alpha)}$ and the conductivities of the plate and the reference medium, and the squared matrices $\mathbf{M}_{p}$ and $\mathbf{M}_{c}$ relative to the Green operators $\mathbf{G}^{p}$ and $\mathbf{G}^{c}$ are computed from the two-point correlation functions $S_{2}^{(\alpha \beta)}$. Additionally, since $\mathbf{G}^{p}$ and $\mathbf{G}^{c}$ are self-adjoint operators (as was mentioned in Sec. III), the matrices $\mathbf{M}_{p}$ and $\mathbf{M}_{c}$ are both symmetric.

From Eq. (39), it is easy to show that the function $\left\langle W_{1}(\tau)\right\rangle$ is stationary when $\mathbf{T}=\mathbf{T}_{s}$ (or equivalently when $\boldsymbol{\tau}=\boldsymbol{\tau}_{s}$ ), with

$$
\mathbf{T}_{s}=\left(\mathbf{M}_{v}+\mathbf{M}_{p}+\mathbf{M}_{c}\right)^{-1} \mathbf{F} .
$$

Correspondingly, the stationary value $\left\langle W_{1}\left(\boldsymbol{\tau}_{s}\right)\right\rangle$ of $\left\langle W_{1}(\boldsymbol{\tau})\right\rangle$ is then given by

$$
\left\langle W_{1}\left(\boldsymbol{\tau}_{s}\right)\right\rangle=\frac{1}{2 N_{3}} \mathbf{T}_{s}^{T} \cdot \mathbf{F} .
$$

Finally, by taking the conductivity of the reference medium such as $c_{0}=\max \left(c_{1}, c_{2}\right)$ [or $\left.c_{0}=\min \left(c_{1}, c_{2}\right)\right]$ and by applying the Hashin-Shtrikman variational principle described above by Eq. (33), i.e.,

$$
W_{\text {eff }}=\frac{1}{2} \mathbf{E}^{0} \cdot \mathbf{C}^{\epsilon} \mathbf{E}^{0}=\frac{1}{2} c_{0} \mathbf{E}^{0} \cdot \mathbf{E}^{0}+\frac{1}{2 N_{3}} \mathbf{T}_{s}^{T} \cdot \mathbf{F},
$$

with an appropriate choice of the macroscopic intensity field $\mathbf{E}^{0}$, the latter equation allows us to obtain the upper (or lower) bound of the effective conductivity components of $\mathbf{C}^{e}$.

A few results will be shown in Sec. VI.

\section{CORRELATION APPROXIMATION FOR THE EFFECTIVE IN-PLANE CONDUCTIVITY}

We apply the formalism of Secs. II and III to estimate the effective behavior of heterogeneous plates. We come back to the third-order approximation (13). Because of the finite ratio between the plate thickness and the size of the inhomogeneities, which leads to the boundary effect, the term $\mathbf{A}_{2}$ would not vanish. We will keep the conductivity $c_{0}$ of the comparison medium as the solution of Eq. (18) (which makes the third-order term vanish in the case of infinite media), and Eq. (13) provides

$$
\left(\frac{1}{c_{0}} \mathbf{C}^{e}-\mathbf{I}\right)^{-1}+\frac{B-1}{d B} \mathbf{I}=-\mathbf{A}_{2},
$$

where $A_{2}$ is defined by (15) and $B$ is determined by (14). Similarly to the computation of the surface integral described 
previously in Sec. IV, the determination of $\mathbf{A}_{2}$ is carried out by writing Eq. (15) in the discretization space. This yields

$$
\mathbf{A}_{2}=\frac{\left(b_{20}-b_{10}\right)^{2}}{N_{3}^{2} B^{2}} \sum_{m, m^{\prime}=1}^{N_{3}} \int_{\omega} \mathbf{h}^{m m^{\prime}}\left(\tilde{\mathbf{x}}^{\prime}\right)\left[S_{22}^{m m^{\prime}}\left(\tilde{\mathbf{x}}^{\prime}\right)-v_{2}^{2}\right] d \tilde{\mathbf{x}}^{\prime},
$$

where $S_{22}^{m m^{\prime}}\left(\tilde{\mathbf{x}}^{\prime}\right)=S_{2}^{(22)}\left(\tilde{\mathbf{x}}^{\prime}, x_{3}(m), x_{3}^{\prime}\left(m^{\prime}\right)\right)$, with $x_{3}(m)$ and $x_{3}^{\prime}\left(m^{\prime}\right)$ being the third coordinates of the $m$ th and $m^{\prime}$ th discretized points, respectively; the second-order tensor $\mathbf{h}^{m m^{\prime}}\left(\tilde{\mathbf{x}}^{\prime}\right)$ is defined as $\mathbf{h}^{m m^{\prime}}\left(\tilde{\mathbf{x}}^{\prime}\right)=\mathbf{h}\left(\tilde{\mathbf{x}}^{\prime}, x_{3}(m), x_{3}^{\prime}\left(m^{\prime}\right)\right)$, with $\mathbf{h}\left(\tilde{\mathbf{x}}^{\prime}, x_{3}(m), x_{3}^{\prime}\left(m^{\prime}\right)\right)$ determined from Eqs. (17) and (6) taking into account the expression of the Green operator $\mathbf{G}(\mathbf{r})$ derived in Sec. III as

$$
\begin{aligned}
\mathbf{h}\left(\tilde{\mathbf{x}}^{\prime}, x_{3}(m), x_{3}^{\prime}\left(m^{\prime}\right)\right) \\
=\frac{1}{d} \delta\left(\tilde{\mathbf{x}}^{\prime}, x_{3}(m)-x_{3}^{\prime}\left(m^{\prime}\right)\right) \mathbf{I}-c_{0}\left[\mathbf{G}^{p}\left(\tilde{\mathbf{x}}^{\prime}, x_{3}(m)-x_{3}^{\prime}\left(m^{\prime}\right)\right)\right. \\
\left.\quad+\mathbf{G}^{c}\left(\tilde{\mathbf{x}}^{\prime}, x_{3}(m), x_{3}^{\prime}\left(m^{\prime}\right)\right)\right] .
\end{aligned}
$$

Next, from Eq. (45), $\mathbf{A}_{2}$ is then computed in Fourier space by employing the FFT with the wave vector $\tilde{\mathbf{k}}=\left(k_{1}, k_{2}\right)$ and taking into account the Parseval theorem. It can be seen immediately from Eq. (46) that the Fourier transform of $\mathbf{h}\left(\tilde{\mathbf{x}}^{\prime}, x_{3}(m), x_{3}^{\prime}\left(m^{\prime}\right)\right)$ takes the following form:

$$
\begin{aligned}
\hat{\mathbf{h}}\left(\tilde{\mathbf{k}}, x_{3}(m), x_{3}^{\prime}\left(m^{\prime}\right)\right)= & \frac{1}{d} \mathbf{I}-c_{0}\left[\hat{\mathbf{G}}^{p}\left(\tilde{\mathbf{k}}, x_{3}(m)-x_{3}^{\prime}\left(m^{\prime}\right)\right)\right. \\
& \left.+\hat{\mathbf{G}}^{c}\left(\tilde{\mathbf{k}}, x_{3}(m), x_{3}^{\prime}\left(m^{\prime}\right)\right)\right],
\end{aligned}
$$

where

$$
\hat{\mathbf{G}}^{p}\left(\tilde{\mathbf{k}}, x_{3}(m)-x_{3}^{\prime}\left(m^{\prime}\right)\right)=\sum_{k_{3}} \hat{\mathbf{G}}^{p}(\mathbf{k}) e^{i k_{3}\left[x_{3}(m)-x_{3}^{\prime}\left(m^{\prime}\right)\right]},
$$

with $\hat{\mathbf{G}}^{p}(\mathbf{k})$ and $\hat{\mathbf{G}}^{c}\left(\tilde{\mathbf{k}}, x_{3}(m), x_{3}^{\prime}\left(m^{\prime}\right)\right)$ determined above in Sec. III.
Note that both sides of Eq. (44) are diagonal matrices due to the choice of the axes, from which-for our purposes-we need only the expression of the in-plane conductivity $c_{e}$ when multiplying both sides of Eq. (44) by $\mathbf{E}^{0}=E_{1}^{0} \mathbf{e}_{1}$.

\section{NUMERICAL APPLICATIONS}

The upper and lower Hashin-Shtrikman bounds established in the previous Secs. IV A and IV B for the effective conductivity of the heterogeneous plate are now numerically illustrated by considering examples in which the spherical particles or circular fibers with the same radius $R$, denoted by phase 2 , are introduced into a host matrix phase, referred to as phase 1. Additionally, to describe the material microgeometry of this heterogeneous plate, the one- and two-point correlation information is provided via the one-point correlation functions $S_{1}^{(\alpha)}(\mathbf{x})$, which are equal to the volume fraction $v_{\alpha}$, and the twopoint correlation functions $S_{2}^{(\alpha \beta)}(\mathbf{x})$, which are assumed to be invariant with respect to any translation and rotation. The analytical and numerical methods to estimate the two-point correlation function for different random material distributions have been studied previously. For more details about these methods, the reader can refer to the excellent book by Torquato [7]

The first example consists of a fiber-reinforced plate containing a random distribution of unidirectional fibers in a matrix phase. The fibers of the plate are assumed to be aligned along the direction $x_{2}$ and have the same circular cross-section of rayon $R$. To describe the distribution of circular fibers in the heterogeneous plate, the two randomly nonoverlapping and overlapping circular disk models are used in [7]. More precisely, in the randomly nonoverlapping disk model, also called the random hard disk model, the two-point correlation functions can be provided by Table 1 in [26]. However, for the overlapping disk model, the two-point correlation functions $S_{2}^{(\alpha \beta)}(r)$ are given explicitly by [7]

$$
S_{2}^{(22)}(r)=v_{2}-v_{1}+S_{2}^{(11)}(r)=v_{2}-S_{2}^{(12)}(r)= \begin{cases}v_{2}^{\frac{2}{\pi}\left[\pi+\frac{r}{2 R}\left(1-\frac{r^{2}}{4 R^{2}}\right)^{1 / 2}-\cos ^{-1}\left(\frac{r}{2 R}\right)\right]} & \text { if } \quad r=\sqrt{x_{1}^{2}+x_{3}^{2}}<2 R, \\ v_{2}^{2} & \text { if } \quad r=\sqrt{x_{1}^{2}+x_{3}^{2}}>2 R .\end{cases}
$$

It is interesting to note that the overlapping disk model is particularly appropriate to describe the porous media in which the voids can be considered as overlapping inclusions with zero conductivity.

As described in Sec. IV B, to obtain the second-order upper and lower bounds of the effective conductivity for both models with randomly nonoverlapping and overlapping distributions of circular disks, we need to calculate the stationary value of $\left\langle W_{1}(\tau)\right\rangle$ provided by Eq. (41). This value can be computed in Fourier space by using FFT. A discretization in the $x_{1}-x_{3}$ plane of the plate is carried out by using the discretized points, whose coordinates are given by

$$
x_{i}=n_{i} \frac{l_{i}}{N_{i}} \quad(i=1,3),
$$

where $\quad N_{i}=2^{p} \quad$ with $\quad p \in \mathrm{N}^{+} \quad$ and $n_{i}=$ $\left[-N_{i} / 2, \ldots, 0, \ldots, N_{i} / 2-1\right]$. The components of the wave vectors are then defined as $k_{i}=2 \pi n_{i} / l_{i}$, where $l_{3}=t$, and $l_{1}$ is large enough compared to the plate thickness and to the size of the inclusions. Practically, all results are the same as soon as $l_{1}$ is greater than $20 R$, which means that for these lengths, the Green tensor for a periodic polarization approximates correctly the Green tensor for a polarization in an infinite plate. So, all computations are effected with $l_{1}=20 R$. With this value for $l_{1}$, a discretization with $N_{1}=N_{3}=2^{7}$ is sufficient to ensure the convergence of the solution.

First, by using the randomly nonoverlapping disk model and by assuming the inclusion phase to be more thermal conducting than the matrix phase with the conductivity ratio of the matrix and inclusions being kept constant with $c_{2} / c_{1}=20$, the upper and lower Hashin-Shtrikman bounds $c_{\text {UHS }} / c_{1}$ and $c_{\text {LHS }} / c_{1}$ that are normalized with respect to $c_{1}$, and the correlation approximation $c_{\mathrm{CA}} / c_{1}$ for the effective in-plane conductivity 


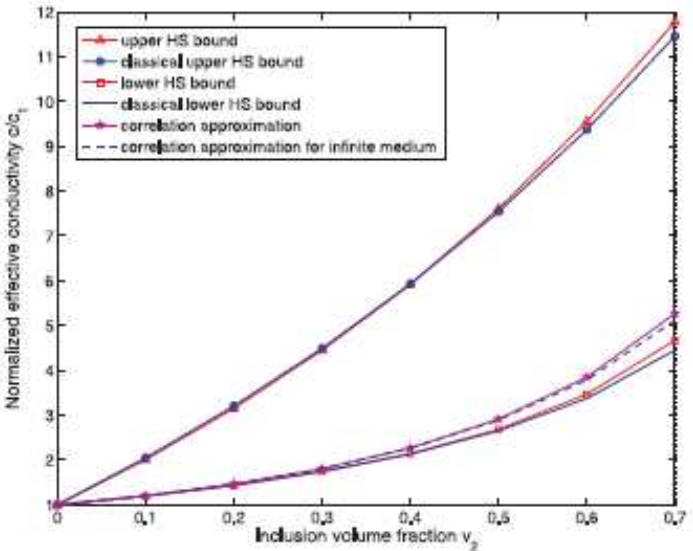

FIG. 2. (Color online) Lower and upper Hashin-Shtrikman bounds that are normalized with respect to $c_{1}$ and the correlation approximation of the effective in-plane conductivity vs the inclusion volume fraction $v_{2}$ with $c_{2} / c_{1}=20, l_{1} / 2 R=10$, and $t / 2 R=2$ (hard disk model).

of the heterogeneous plate that is nomalized with respect to $c_{1}$, are plotted versus the volume fraction $v_{2}$ of the inclusion phase in Fig. 2 with $l_{1} / 2 R=10$ and $t / 2 R=2$. These values for $c_{\mathrm{UHS}} / c_{1}, c_{\mathrm{LHS}} / c_{1}$, and $c_{\mathrm{CA}} / c_{1}$ are also compared in Fig. 2 with the corresponding values for an infinite medium. Additionally, compared to the classical upper and lower Hashin-Shtrikman bounds and the correlation approximation for an infinite medium, we show numerically that the relative differences obtained for the bounds and correlation approximation of the effective in-plane conductivity with $l_{1} / 2 R=t / 2 R=10$ are less than $0.6 \%$ (while those of Fig. 2 are about $3 \%$ ). These results allow us to conclude that, as soon as $t / 2 R=10$, the plate does not display any boundary effect.

Figure 2 shows that when the size of the inhomogeneities is compared to the thickness of the plate $\left(l_{1} / 2 R=10\right.$ and $t / 2 R=2$ ), the upper and lower Hashin-Shtrikman bounds for the effective in-plane conductivity obtained from the theory established in the previous section (Secs. IV A and IV B) appear lower (smaller) than the respective classical upper and lower Hashin-Shtrikman bounds. As expected, we can observe also from Fig. 2 that the correlation approximation values obtained for the effective in-plane conductivity are well situated between the upper and lower Hashin-Shtrikman bounds. Similar results are observed in Fig. 3 for the randomly overlapping disk model, with the inclusion phase being less thermally conducting than the matrix phase $\left(c_{2} / c_{1}=1 / 20\right)$. Moreover, it can be seen from Figs. 2 and 3 that the values obtained for the effective in-plane conductivity are close either to the lower Hashin-Shtrikman bound for the case in which the inclusion phase is more thermally conducting than the matrix phase, or to the upper Hashin-Shtrikman bound for the opposite case. Figures 4 and 5 address the two extreme cases with superconducting inclusions $\left(c_{2} / c_{1}=\infty\right)$ and perfectly insulating inclusions $\left(c_{2} / c_{1}=0\right)$. Figure 4 displays the lower Hashin-Shtrikman bound and correlation approximation of the effective in-plane conductivity that is normalized with respect

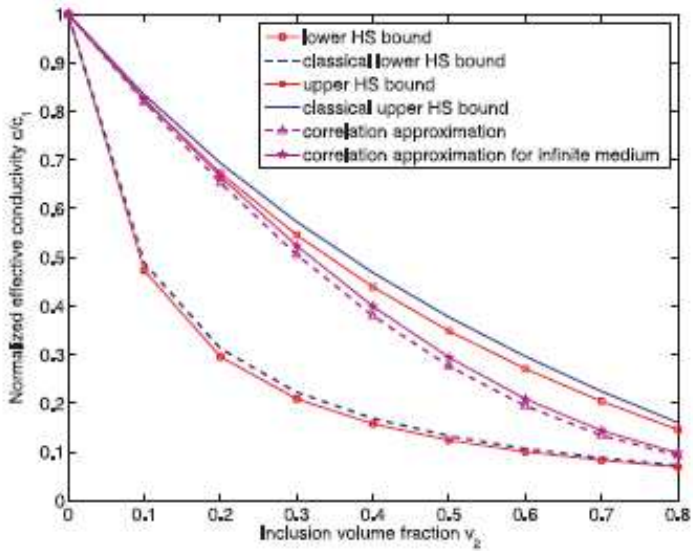

FIG. 3. (Color online) Lower and upper Hashin-Shtrikman bounds that are normalized with respect to $c_{1}$ and the correlation approximation of the effective in-plane conductivity vs the inclusion volume fraction $v_{2}$ with $c_{2} / c_{1}=1 / 20, l_{1} / 2 R=10$, and $t / 2 R=2$ (overlapping disk model).

to the matrix conductivity $c_{1}$ versus the inclusion volume fraction $v_{2}$ with the random overlapping disk model. Figure 5 displays the upper Hashin-Shtrikman bound and correlation approximation of the effective in-plane conductivity that is normalized with respect to the matrix conductivity $c_{1}$ versus the inclusion volume fraction $v_{2}$ with the random overlapping disk model.

Next, to understand more details about the size dependence of the Hashin-Shtrikman bounds, we show in Figs. 6, 7, 8, and 9 the upper and lower bounds that are normalized with respect to $c_{1}$, as well as the correlation approximation for the effective in-plane conductivity in terms of the inclusion volume fraction $v_{2}$ with different values of the ratio between

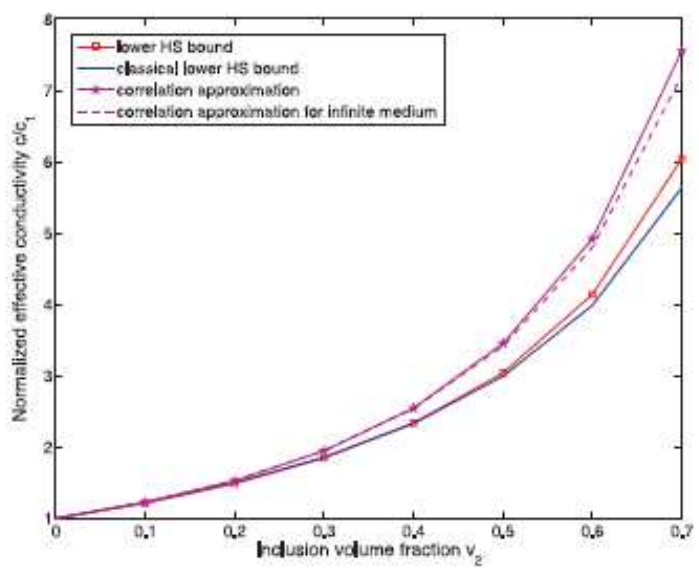

FIG. 4. (Color online) Lower Hashin-Shtrikman bound that is normalized with respect to $c_{1}$ and the correlation approximation of the effective in-plane conductivity vs the inclusion volume fraction $v_{2}$ with $c_{2} / c_{1}=\infty, l_{1} / 2 R=10$, and $t / 2 R=2$ (hard disk model). 
the plate thickness and inhomogeneity size $t / 2 R$ for the two extreme cases. It can be seen from Figs. 6, 7, 8, and 9 that the size dependence of Hashin-Shtrikman bounds is more pronounced as the ratio $t / 2 R$ decreases. The upper and lower Hashin-Shtrikman bounds that are normalized with respect to $c_{1}$ and the correlation approximation for the effective in-plane conductivity are presented in terms of the ratio between the plate thickness and the size of the inhomogeneities, $t / 2 R$, in Figs. 10 and 11 , respectively, while the volume fraction of the inhomogeneity phase is kept constant with $v_{2}=0.7$ for the superconducting inclusion case and $v_{2}=0.8$ for perfectly insulated inclusion case.

In the second example, the plate is now reinforced by identically spherical particles. The two randomly nonoverlapping and overlapping spheres models are used to describe the distribution of spherical particles in the heterogeneous plate. More precisely, in the randomly nonoverlapping sphere model, also called the random hard sphere model, the two-point correlation functions can be expressed as $[27,28]$

$$
S_{2}^{(\alpha \beta)}\left(\mathbf{x}-\mathbf{x}_{2}^{(\alpha \beta)}\right)(r)=v_{\alpha} v_{\beta}+v_{\alpha}\left(\delta_{\alpha \beta}-v_{\beta}\right) h(r),
$$

where $r$ denotes the distance between two points $\mathbf{x}$ and $\mathbf{x}^{\prime}, \delta_{\alpha \beta}$ is the Kronecker symbol, and $h(r)$ is an exponential function defined as follows $[26,29]$ :

$$
h(r)=e^{-\frac{r}{a}} .
$$

Here, the coefficient $a$ depends on both the radius $R$ of spherical inclusions and the volume fraction $v_{1}$ of the matrix phase,

$$
a^{2}=R^{2} \frac{5 b_{1} v_{1} d_{1}+2 g_{1}\left[d_{1}\left(2+v_{1}\right)-v_{1}\left(\tilde{v}_{1} / v_{1}\right)^{2 / 3}\left(9-d_{1}+\tilde{v}_{1}^{2}\right)\right]}{10 g_{1}\left(1-v_{1}\right) d_{1}},
$$

where

$$
\begin{gathered}
\tilde{v}_{1}=v_{1}-\frac{1}{16} v_{1}^{2}, \quad b_{1}=\frac{3}{2} \frac{\tilde{v}_{1}^{2}\left(1-0.7117 \tilde{v}_{1}-0.114 \tilde{v}_{1}^{2}\right)}{\left(1-\tilde{v}_{1}\right)^{4}}, \\
g_{1}=12 b_{1} \frac{\left(1-\tilde{v}_{1}\right)^{2}}{\tilde{v}_{1}\left(2+\tilde{v}_{1}\right)}, \quad d_{1}=1+2 \tilde{v}_{1} .
\end{gathered}
$$

In the overlapping sphere model, the two-point correlation functions $S_{2}^{(\alpha \beta)}(r)$ are provided by $[7,30]$

$$
\begin{aligned}
S_{2}^{(22)}(r) & =v_{2}-v_{1}+S_{2}^{(11)}(r)=v_{2}-S_{2}^{(12)}(r) \\
& =\left\{\begin{array}{lll}
v_{2}^{\left(1+\frac{3 r}{4 R}-\frac{r^{3}}{16 R^{3}}\right)} & \text { if } & r<2 R, \\
v_{2}^{2} & \text { if } & r>2 R .
\end{array}\right.
\end{aligned}
$$

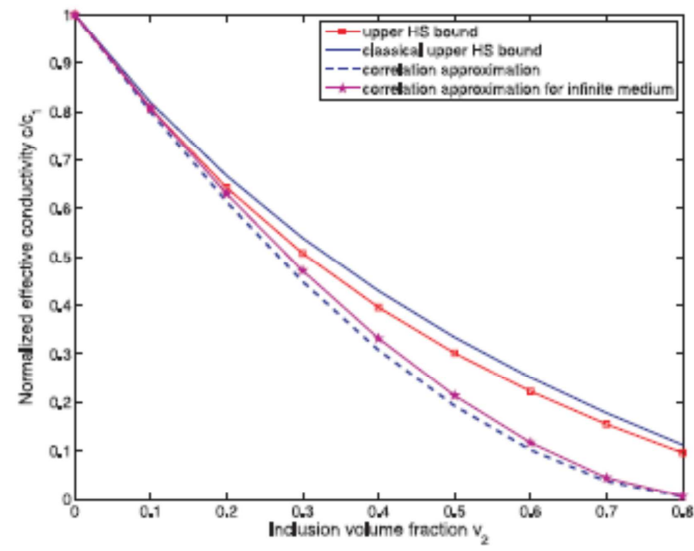

FIG. 5. (Color online) Upper Hashin-Shtrikman bound that is normalized with respect to $c_{1}$ and the correlation approximation of the effective in-plane conductivity vs the inclusion volume fraction $v_{2}$ with $c_{2} / c_{1}=0, l_{1} / 2 R=10$, and $t / 2 R=2$ (overlapping disk model).
As before, to calculate the stationary value of $\left\langle W_{1}(\tau)\right\rangle$ provided by Eq. (41) in the Fourier space by using FFT, a discretization in the three-dimensional space of the plate is necessary. The coordinates of the discretized points are then given by

$$
x_{i}=n_{i} \frac{l_{i}}{N_{i}} \quad(i=1,2,3),
$$

where $N_{i}=2^{p} \quad$ with $\quad p \in \mathbf{N}^{+}$and $n_{i}=$ $\left[-N_{i} / 2, \ldots, 0, \ldots, N_{i} / 2-1\right]$. The components of the wave vectors are then defined as $k_{i}=2 \pi n_{i} / l_{i}$. In our computations for the three-dimensional case, a discretization with $N_{1}=N_{2}=N_{3}=2^{6}$ is enough to ensure the convergence of the solution. $l_{3}$ is still equal to $t$ while $l_{1}$ and $l_{2}$ are set to $l_{1}=l_{2}=20 \mathrm{R}$ for the reasons given previously in the $2 \mathrm{D}$ case.

By using the randomly nonoverlapping sphere model and by assuming the inclusion phase to be more thermally conducting than the matrix phase with the conductivity ratio

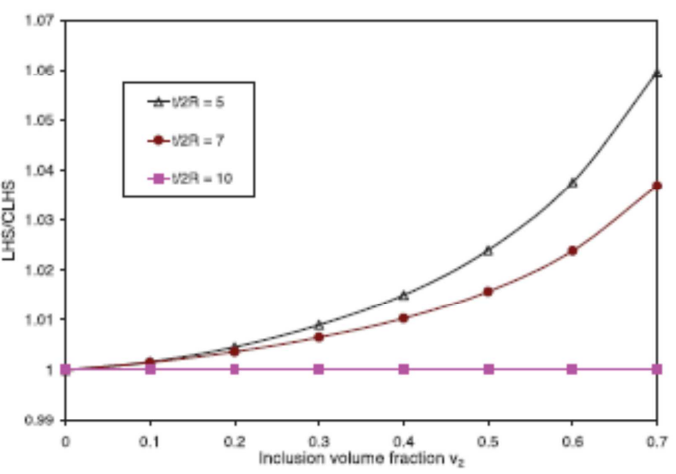

FIG. 6. (Color online) Lower Hashin-Shtrikman bound of inplane effective conductivity that is normalized with respect to the corresponding values for an infinite medium vs the inclusion volume fraction $v_{2}$ with $c_{2} / c_{1}=\infty$ and $l_{1} / 2 R=10$ (hard disk model). 


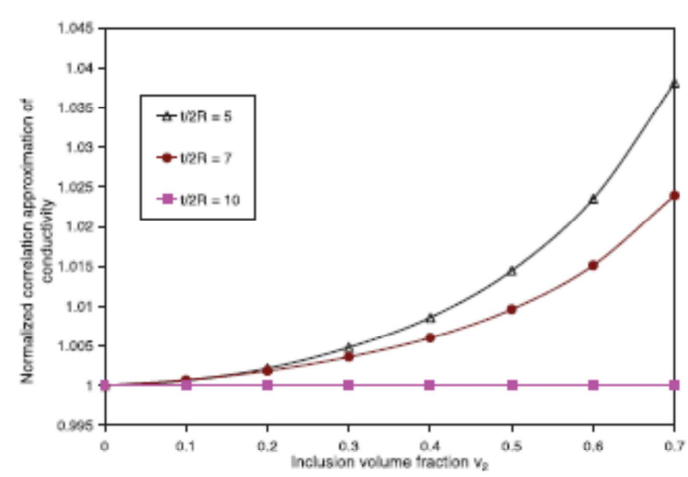

FIG. 7. (Color online) Correlation approximation of in-plane effective conductivity that is normalized with respect to the corresponding values for an infinite medium vs the inclusion volume fraction $v_{2}$ with $c_{2} / c_{1}=\infty$ and $l_{1} / 2 R=10$ (hard disk model).

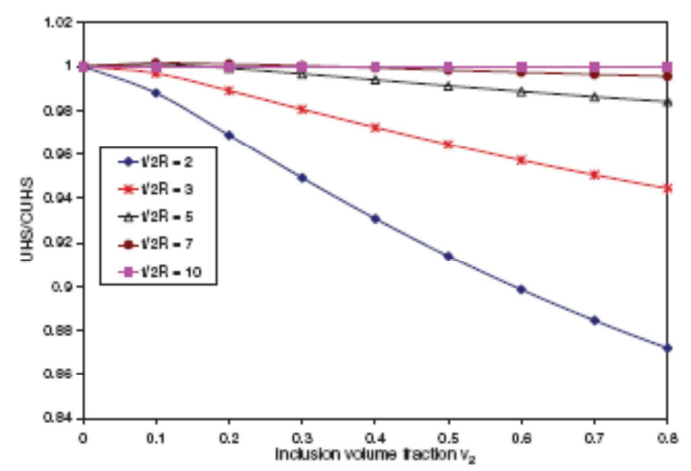

FIG. 8. (Color online) Upper Hashin-Shtrikman bound of inplane effective conductivity that is normalized with respect to the corresponding values for an infinite medium vs the inclusion volume fraction $v_{2}$ with $c_{2} / c_{1}=0$ and $l_{1} / 2 R=10$ (overlapping disk model).

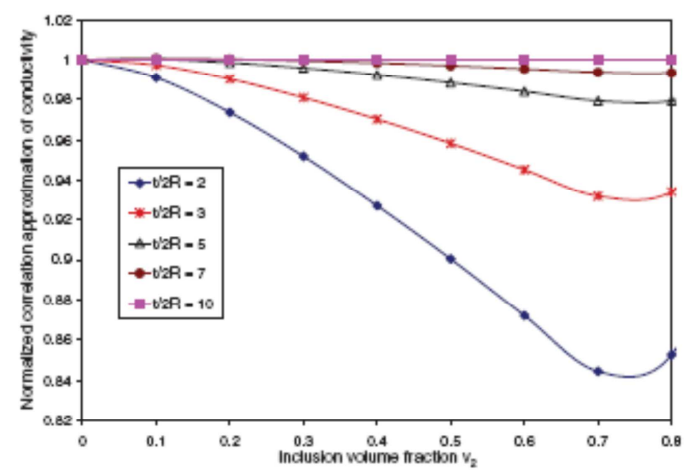

FIG. 9. (Color online) Correlation approximation of in-plane effective conductivity that is normalized with respect to the corresponding values for an infinite medium vs the inclusion volume fraction $v_{2}$ with $c_{2} / c_{1}=0$ and $l_{1} / 2 R=10$ (overlapping disk model)

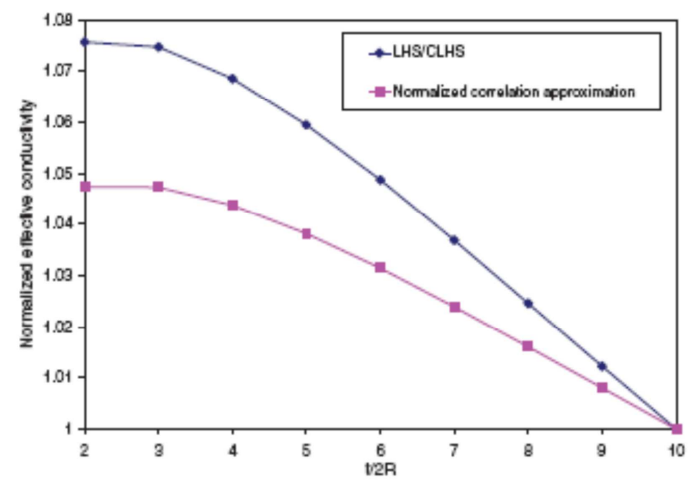

FIG. 10. (Color online) Lower Hashin-Shtrikman bound that is normalized with respect to corresponding values for an infinite medium and the correlation approximation of effective in-plane conductivity vs the ratio $t / 2 R$ with $v_{2}=0.7, c_{2} / c_{1}=\infty$, and $l_{1} / 2 R=10$ (hard disk model).

of the matrix and inclusions being kept constant, $c_{2} / c_{1}=20$, the normalized upper and lower Hashin-Shtrikman bounds, $c_{U H S} / c_{1}$ and $c_{\mathrm{LHS}} / c_{1}$, as well as the normalized correlation approximation, $c_{\mathrm{CA}} / c_{1}$, of the effective in-plane conductivity of the heterogeneous plate are plotted versus the volume fraction $v_{2}$ of the inclusion phase in Fig. 12 with $l_{1} / 2 R=$ $l_{2} / 2 R=10$ and $t / 2 R=2$. These nomalized bounds $c_{\text {UHS }} / c_{1}$ and $c_{\mathrm{LHS}} / c_{1}$ and normalized correlation approximation $c_{\mathrm{CA}} / c_{1}$ are also compared in Fig. 12 with the normalized classical upper and lower Hashin-Shtrikman bounds, $c_{\mathrm{CUHS}} / c_{1}$ and $c_{\mathrm{CLHS}} / c_{1}$, and the normalized correlation approximation for an infinite medium. It is seen from Fig. 12 that the correlation approximation values obtained for the effective in-plane conductivity are well located between the upper and lower Hashin-Shtrikman bounds. Moreover, we observe also from Fig. 12 that the estimation values obtained for the effective

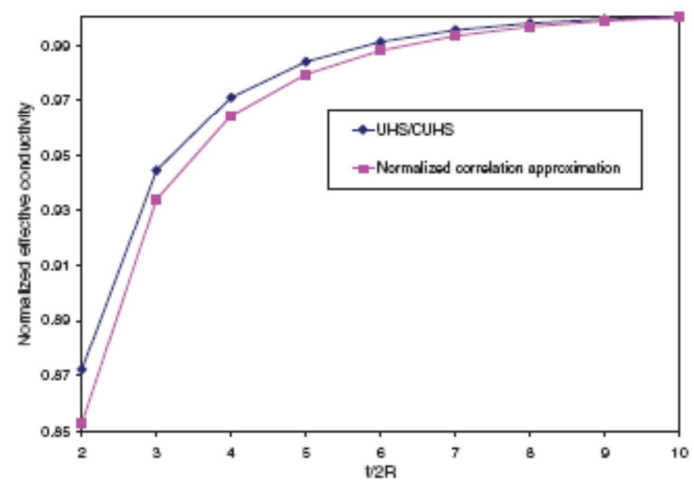

FIG. 11. (Color online) Upper Hashin-Shtrikman bound that is normalized with respect to corresponding values for an infinite medium and the correlation approximation of effective in-plane conductivity vs the ratio $t / 2 R$ with $v_{2}=0.8, c_{2} / c_{1}=0$, and $l_{1} / 2 R=10$ (overlapping disk model). 


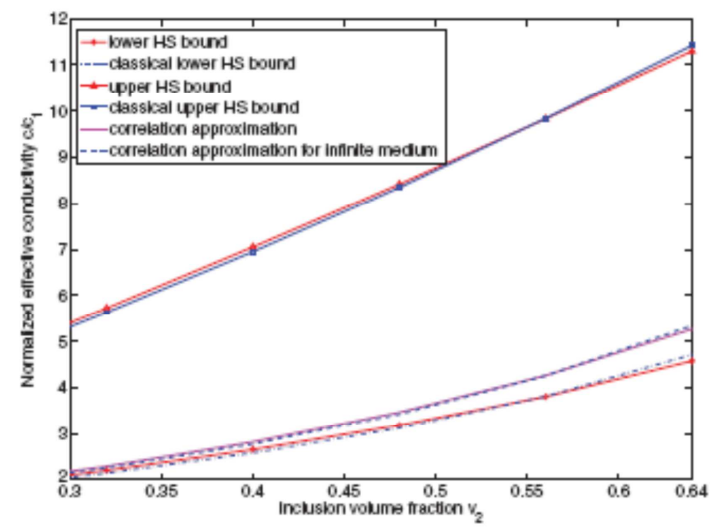

FIG. 12. (Color online) Lower and upper Hashin-Shtrikman bounds that are normalized with respect to $c_{1}$ and the correlation approximation of the effective in-plane conductivity vs the inclusion volume fraction $v_{2}$ with $c_{2} / c_{1}=20, l_{1} / 2 R=l_{2} / 2 R=10$, and $t / 2 R=2$ (hard sphere model).

in-plane conductivity for the hard sphere model with higher conducting inclusions are close to the lower Hashin-Shtrikman bound. As can be seen in Fig. 12, there is a size effect on the upper and lower Hashin-Shtrikman bounds as well as on the correlation approximation for the effective in-plane conductivity of the heterogeneous plate where the size of the inhomogeneities is compared to the thickness of the plate for $t / 2 R=2$.

Furthermore, to study more details about the size dependence of the Hashin-Shtrikman bounds as well as the correlation approximation, we present in Fig. 13 the upper and lower bounds that are normalized with respect to the corresponding values for an infinite medium, and the correlation approximation for the effective in-plane conductivity in terms of the ratio between the thickness of the plate and the size of

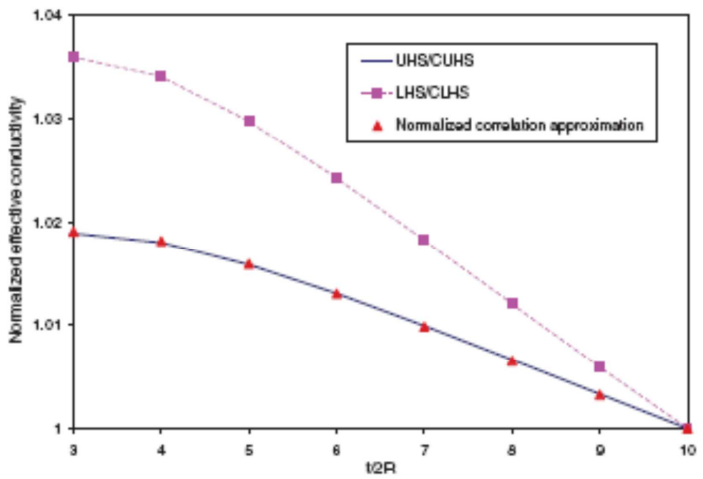

FIG. 13. (Color online) Lower and upper Hashin-Shtrikman bounds that are normalized with respect to the corresponding values for an infinite medium and the correlation approximation of effective in-plane conductivity vs the ratio $t / 2 R$ with $v_{2}=0.4, c_{2} / c_{1}=20$, and $l_{1} / 2 R=l_{2} / 2 R=10$ (hard sphere model).

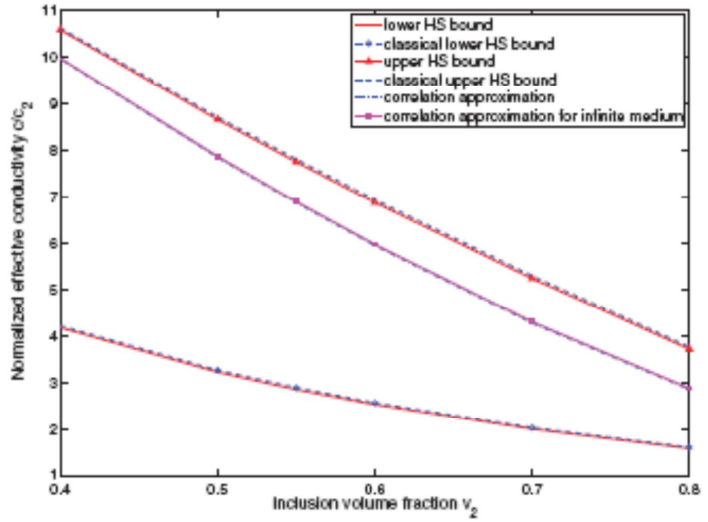

FIG. 14. (Color online) Lower and upper Hashin-Shtrikman bounds that are normalized with respect to $c_{2}$ and the correlation approximation of the effective in-plane conductivity vs the inclusion volume fraction $v_{2}$ with $c_{2} / c_{1}=1 / 20, l_{1} / 2 R=l_{2} / 2 R=10$, and $t / 2 R=2$ (overlapping sphere model).

inhomogeneity, $t / 2 R$, for a given value of the inclusion volume fraction $c_{2}=0.4$. It can be seen that the size dependence of the Hashin-Shtrikman bounds and the correlation approximation is more pronounced as the ratio $t / 2 R$ decreases.

For the overlapping sphere model, by setting the inclusion phase to be less conducting than the matrix phase with $c_{2} / c_{1}=1 / 20$, the normalized upper and lower bounds and the correlation approximation of the effective in-plane conductivity for a thin heterogeneous plate with $t / 2 R=2$ are plotted in Fig. 14 and compared with their counterpart values for an infinite medium. Compared with the relevant results for the nonoverlapping sphere model, it is shown in Fig. 14 that the size dependence of the upper and lower bounds and the correlation approximation of the effective

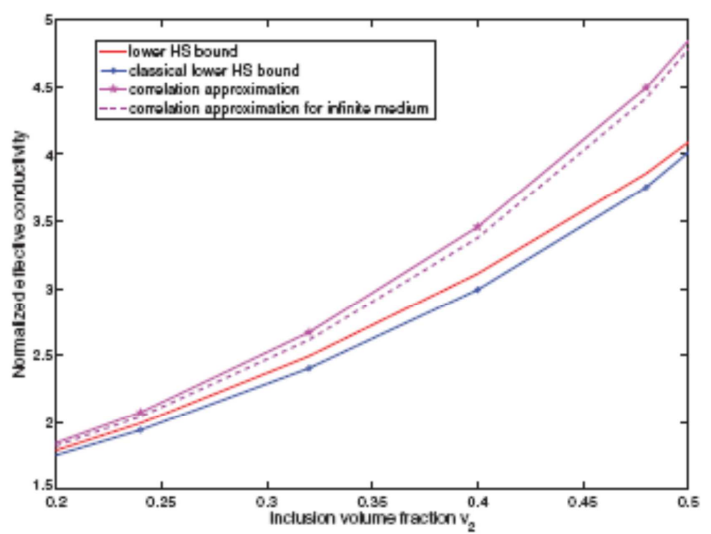

FIG. 15. (Color online) Lower Hashin-Shtrikman bound that is normalized with respect to $c_{1}$ and the correlation approximation of the effective in-plane conductivity vs the inclusion volume fraction $v_{2}$ with $c_{2} / c_{1}=\infty, l_{1} / 2 R=l_{2} / 2 R=10$, and $t / 2 R=2$ (hard sphere model). 


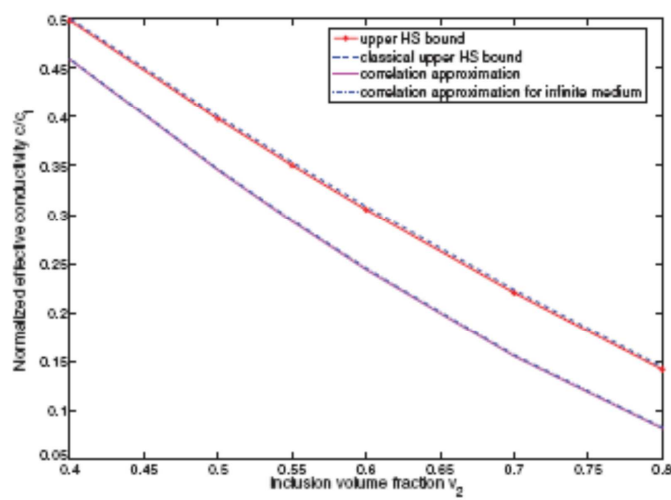

FIG. 16. (Color online) Upper Hashin-Shtrikman bound that is normalized with respect to $c_{1}$ and the correlation approximation of the effective in-plane conductivity vs the inclusion volume fraction $v_{2}$ with $c_{2} / c_{1}=0, l_{1} / 2 R=l_{2} / 2 R=10$, and $t / 2 R=2$ (overlapping sphere model)

in-plane conductivity is much smaller (less than 1\%). Moreover, because the matrix phase is more conducting than the inclusion phase, the correlation approximation of effective in-plane conductivity obtained in this case is then nearer to the upper Hashin-Shtrikman bound

Next, we consider the two extreme cases in which the inclusion phase is assumed to be superconducting, i.e., $c_{2} / c_{1}=$ $\infty$, and perfectly insulating, i.e., $c_{2} / c_{1}=0$. More precisely, we present in Fig. 15 the lower Hashin-Shtrikman bound that is normalized with respect to $c_{1}$ and the correlation estimation of the effective in-plane conductivity in terms of the inclusion volume fraction according to the hard sphere model with $l_{1} / 2 R=l_{2} / 2 R=10$ and $t / 2 R=2$. Comparing Fig. 12 with Fig. 15, we notice that the size dependence of the lower Hashin-Shtrikman bound and the correlation estimation

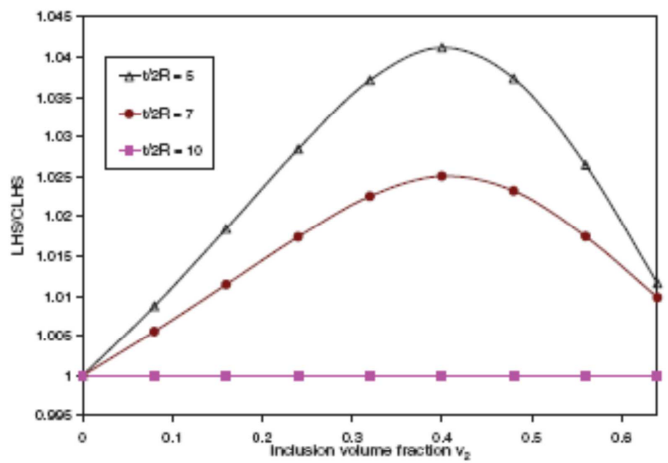

FIG. 17. (Color online) Lower Hashin-Shtrikman bound of in-plane effective conductivity that is normalized with respect to the corresponding values for an infinite medium vs the inclusion volume fraction $v_{2}$ with $c_{2} / c_{1}=\infty$ and $l_{1} / 2 R=l_{2} / 2 R=10$ (hard sphere model).

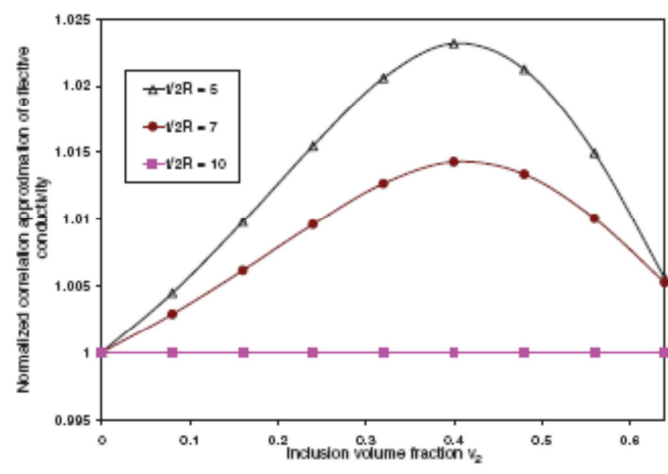

FIG. 18. (Color online) Correlation approximation of in-plane effective conductivity that is normalized with respect to the corresponding values for an infinite medium vs the inclusion volume fraction $v_{2}$ with $c_{2} / c_{1}=\infty$ and $l_{1} / 2 R=l_{2} / 2 R=10$ (hard sphere model).

of the effective in-plane conductivity for the superconducting inclusion case is more pronounced than in the first example with $c_{2} / c_{1}=20$. Concerning the second extreme case with perfectly insulating inclusions, we plot in Fig. 16 the upper Hashin-Shtrikman bound that is normalized with respect to $c_{2}$ and the correlation estimation of the effective in-plane conductivity by applying the overlapping sphere model. It can be seen again from Fig. 16 that the size effect of the upper Hashin-Shtrikman bound and the correlation estimation of the effective in-plane conductivity with the overlapping sphere model is very small in spite of the extreme case with the ratio $c_{2} / c_{1}=0$. As before, the size dependences of the lower Hashin-Shtrikman bound and the correlation approximation of the effective in-plane conductivity are then presented in Figs. 17 and 18 in terms of the inclusion volume fraction $v_{2}$ and according to different values of the ratio $t / 2 R$ in the superconducting inclusion case. Figures 17 and 18 show

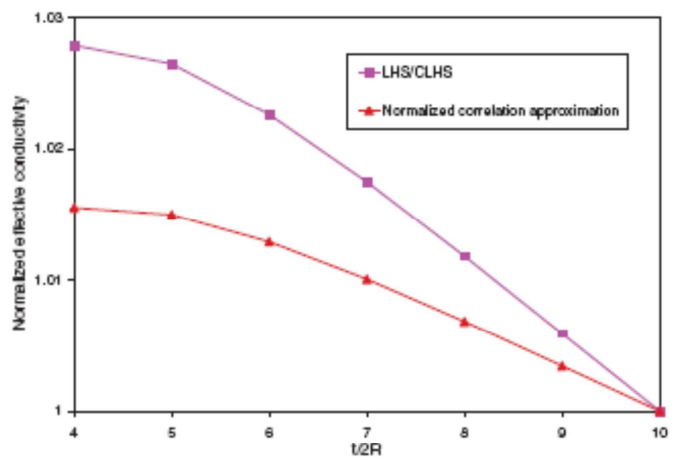

FIG. 19. (Color online) Lower Hashin-Shtrikman bound that is normalized with respect to corresponding values for an infinite medium and the correlation approximation of effective in-plane conductivity vs the ratio $t / 2 R$ with $v_{2}=0.56, c_{2} / c_{1}=\infty$, and $l_{1} / 2 R=l_{2} / 2 R=10$ (hard sphere model). 
that the size dependences become more important as the ratio $t / 2 R$ decreases. Similar results are also observed in Fig. 19, in which the normalized lower Hashin-Shtrikman bound and the correlation approximation of the effective in-plane conductivity are plotted in terms of the ratio $t / 2 R$ in the superconducting inclusion case with $v_{2}=0.56$.

\section{CONCLUSION}

Usually, effective properties of materials do not depend on the size of heterogeneities. However, in the case of plates, a size effect appears when the size of the heterogeneities is no longer negligible compared to the plate thickness. This effect was studied in the case of the in-plane conductivity of heterogeneous plates by producing an extension for plates of Hashin-Shtrikman bounds and of approximations accounting for third-order correlations. Results are obtained for 2D distributions of circular inclusions and 3D distributions of spheres. They show that the size effect leads to decreasing the effective conductivity and can reach more than $10 \%$ both for the third correlation approximation and Hashin-Shtrikman bounds in the $2 \mathrm{D}$ case when inclusions have a radius that becomes half the plate thickness. This effect becomes significantly smaller (less than $5 \%$ ) in the 3D case. The tendencies (increasing or decreasing) of the effective conductivity of the heterogeneous plate compared to those for the infinite medium observed for the upper and lower bounds and the correlation approximation over the ranges of parameters appear to be in agreement. In this work, we examined the insulation boundary conditions on the free boundaries of the heterogeneous plate. It should be interesting to study the thin plate under other boundary conditions.

\section{ACKNOWLEDGMENTS}

We thank the Université Paris-Est Marne-la-Vallée and Vietnam's Nafosted for providing support for D.C.P

\section{APPENDIX: THE EXPRESSIONS FOR THE GREEN} OPERATORS GP AND G ${ }^{e}$ IN EQ. (28)

We will show in this appendix that the expressions of the solution fields in (24) and (26) as well as the expressions of $\mathbf{G}^{P}, \mathbf{G}^{c}$, and $\mathbf{G}$ can be obtained explicitly in the Fourier space by using fast Fourier transform (FFT) [31,32]. In fact, for any function $f(\mathbf{x})$ that is 3 D-periodic and $g(\mathbf{x})$ that is $2 \mathrm{D}$-periodic in the $x_{1}-x_{2}$ plane, the expansions into Fourier series of $f(\mathbf{x})$ and $g(\mathbf{x})$ are given by

$$
f(\mathbf{x})=\sum_{\mathbf{k}} \hat{f}(\mathbf{k}) e^{i \mathbf{k} \cdot \mathbf{x}}, \quad g(\mathbf{x})=\sum_{\mathbf{k}} \hat{g}\left(\tilde{\mathbf{k}}, x_{3}\right) e^{i \hat{\mathbf{k}} \cdot \mathbf{x}},
$$

where $i=\sqrt{-1} ; \tilde{\mathbf{k}}=\left(k_{1}, k_{2}\right)$ and $\mathbf{k}=\left(k_{1}, k_{2}, k_{3}\right)$ are the discrete wave vectors arranged along a discrete lattice with period $2 \pi / l_{i}\left(l_{3}=t\right)$ along the $x_{i}$ direction; the coefficients of the Fourier series $\hat{f}(\mathbf{k})$ and $\hat{g}\left(\tilde{\mathbf{k}}, x_{3}\right)$ are calculated by FFT.

According to this method, the temperature solution fields $T^{p}(\mathbf{x})$ and $T^{c}(\mathbf{x})$ of the boundary-value problems (24) and (26) are expanded into the Fourier series by

$$
T^{p}(\mathbf{x})=\sum_{\mathbf{k}} \hat{T}^{P}(\mathbf{k}) e^{i \mathbf{k} \cdot \mathbf{x}}, \quad T^{c}(\mathbf{x})=\sum_{\mathbf{k}} \hat{T}^{c}\left(\tilde{\mathbf{k}}, x_{3}\right) e^{i \mathrm{k} \cdot \mathbf{x}} .
$$

The resulting intensity fields are given by

$$
\mathbf{E}^{p}(\mathbf{x})=\sum_{\mathbf{k}} \hat{\mathbf{E}}^{p}(\mathbf{k}) e^{i \mathbf{k} \cdot \mathbf{x}}, \quad \mathbf{E}^{c}(\mathbf{x})=\sum_{\mathbf{k}} \hat{\mathbf{E}}^{c}\left(\tilde{\mathbf{k}}, x_{3}\right) e^{i \mathbf{k} \cdot \mathbf{x}},
$$

where $\hat{\mathbf{E}}^{P}(\mathbf{k})$ and $\hat{\mathbf{E}}^{c}\left(\tilde{\mathbf{k}}, x_{3}\right)$ are calculated from the temperature fields $\hat{T}^{P}(\mathbf{k})$ and $\hat{T}^{c}\left(\tilde{\mathbf{k}}, x_{3}\right)$ by

$$
\hat{\mathbf{E}}^{p}(\mathbf{k})=-\nabla^{p} \otimes \hat{T}^{p}(\mathbf{k}), \quad \hat{\mathbf{E}}^{c}\left(\tilde{\mathbf{k}}, x_{3}\right)=-\nabla^{c} \otimes \hat{T}^{c}\left(\tilde{\mathbf{k}}, x_{3}\right),
$$

where $\nabla^{p}$ and $\nabla^{c}$ are two operators defined by $\nabla^{P}=$ $\left[\begin{array}{lll}i k_{1} & i k_{2} & i k_{3}\end{array}\right]^{T}$ and $\nabla^{c}=\left[\begin{array}{lll}i k_{1} & i k_{2} & \partial_{3}\end{array}\right]^{T}$, and the operator $\partial$ denotes the partial differentiation with respect to the coordinate subscript that follows.

Next, by writing the polarization field $\mathbf{p}(\mathbf{x})$ into the Fourier series as

$$
\mathbf{p}(\mathbf{x})=\sum_{\mathbf{k}} \hat{\mathbf{p}}(\mathbf{k}) e^{i \mathbf{k} \cdot \mathbf{x}},
$$

the heat flux fields obtained from the Fourier law as

$$
\hat{\mathbf{q}}^{p}(\mathbf{k})=c_{0} \hat{\mathbf{E}}^{p}(\mathbf{k})+\hat{\mathbf{p}}(\mathbf{k}), \quad \hat{\mathbf{q}}^{c}\left(\tilde{\mathbf{k}}, x_{3}\right)=c_{0} \hat{\mathbf{E}}^{c}\left(\tilde{\mathbf{k}}, x_{3}\right),
$$

must verify, for the stationary thermal conduction case without a heat source, the following energy conservation equation:

$$
\begin{aligned}
c_{0} k^{2} \hat{T}^{p}(\mathbf{k})+i \mathbf{k} \cdot \hat{\mathbf{p}}(\mathbf{k}) & =0, \\
c_{0} s^{2} \hat{T}^{c}\left(\tilde{\mathbf{k}}, x_{3}\right)-c_{0} \partial_{33}^{2} \hat{T}^{c}\left(\tilde{\mathbf{k}}, x_{3}\right) & =0,
\end{aligned}
$$

with $k=\left(k_{1}^{2}+k_{2}^{2}+k_{3}^{2}\right)^{1 / 2}$ and $s=\left(k_{1}^{2}+k_{2}^{2}\right)^{1 / 2}$. The expressions of the temperature solution fields $\hat{T}^{P}(\mathbf{k})$ and $\hat{T}^{c}\left(\tilde{\mathbf{k}}, x_{3}\right)$ obtained from (A7) take the form

$$
\begin{gathered}
\hat{T}^{p}(\mathbf{k})=-\frac{i \mathbf{k} \cdot \hat{\mathbf{p}}(\mathbf{k})}{c_{0} k^{2}}, \quad \forall \mathbf{k} \neq \mathbf{0}, \\
\hat{T}^{c}\left(\tilde{\mathbf{k}}, x_{3}\right)=a^{+} e^{s x_{3}}+a^{-} e^{-s x_{3}}, \quad \forall \tilde{\mathbf{k}} \neq \mathbf{0} .
\end{gathered}
$$

In the preceding equation, $a^{ \pm}$are two constants to be determined via the vector $\mathbf{a}(\tilde{\mathbf{k}})=\left(a^{+}, a^{-}\right)^{T}$ from the boundary conditions on $\partial Y^{ \pm}$,

$$
\hat{q}_{3}^{c}\left(\tilde{\mathbf{k}}, x_{3}= \pm \frac{t}{2}\right)=-\sum_{k_{3}} \hat{q}_{3}^{p}(\mathbf{k}) e^{ \pm \frac{i_{3} r}{2}} .
$$

This yields

$$
\mathbf{a}(\tilde{\mathbf{k}})=\mathbf{K}^{-1}(\tilde{\mathbf{k}}) \mathbf{Q}(\tilde{\mathbf{k}}), \quad \forall \tilde{\mathbf{k}} \neq \mathbf{0},
$$

where

$$
\begin{gathered}
\mathbf{K}(\tilde{\mathbf{k}})=s c_{0}\left[\begin{array}{ll}
-e^{\frac{\pi t}{2}} & e^{-\frac{\pi t}{2}} \\
-e^{-\frac{n t}{2}} & e^{\frac{\pi t}{2}}
\end{array}\right], \\
\mathbf{Q}(\tilde{\mathbf{k}})=\left[\begin{array}{c}
Q^{+} \\
Q^{-}
\end{array}\right]=-\left[\begin{array}{l}
\sum_{k_{3}} \hat{q}_{3}^{P}(\mathbf{k}) e^{\frac{i_{3} t}{2}} \\
\sum_{k_{3}} \hat{q}_{3}^{P}(\mathbf{k}) e^{-\frac{i_{3} t}{2}}
\end{array}\right] .
\end{gathered}
$$


Substituting (A9) into (A4) together with (A11)-(A13) leads to

$$
\begin{aligned}
\hat{\mathbf{E}}^{p}(\mathbf{k}) & =-\left(\frac{\mathbf{k} \otimes \mathbf{k}}{c_{0} k^{2}}\right) \hat{\mathbf{p}}(\mathbf{k}), \quad \forall \mathbf{k} \neq \mathbf{0}, \\
\hat{\mathbf{E}}^{c}\left(\tilde{\mathbf{k}}, x_{3}\right) & =\mathbf{P}\left(\tilde{\mathbf{k}}, x_{3}\right) \mathbf{K}^{-1}(\tilde{\mathbf{k}}) \mathbf{Q}(\tilde{\mathbf{k}}), \forall \tilde{\mathbf{k}} \neq \mathbf{0},
\end{aligned}
$$

with

$$
\mathbf{P}\left(\tilde{\mathbf{k}}, x_{3}\right)=\left[\begin{array}{ll}
-i k_{1} e^{s x_{3}} & -i k_{1} e^{-s x_{3}} \\
-i k_{2} e^{s x_{3}} & -i k_{2} e^{-s x_{3}} \\
-s e^{s x_{3}} & s e^{-s x_{3}}
\end{array}\right] .
$$

By writing (25) in the Fourier space

$$
\hat{\mathbf{E}}^{P}(\mathbf{k})=-\hat{\mathbf{G}}^{p}(\mathbf{k}) \hat{\mathbf{p}}^{p}(\mathbf{k}),
$$

and by comparing (A17) with (A14), we obtain the Fourier transform of the Green operator $\mathrm{G}^{P}$ such as

$$
\hat{\mathbf{G}}^{p}(\mathbf{k})=\left(\frac{\mathbf{k} \otimes \mathbf{k}}{c_{0} k^{2}}\right), \quad \forall \mathbf{k} \neq \mathbf{0} .
$$

Moreover, owing to the fact that

$$
Q^{ \pm}(\tilde{\mathbf{k}})=-\sum_{k_{3}} \hat{q}_{3}^{p}(\mathbf{k}) e^{ \pm \frac{i_{3} t}{2}}=-\sum_{k_{3}} \Delta_{3 m}(\mathbf{k}) \hat{p}_{m}(\mathbf{k}) e^{ \pm \frac{i_{3} t}{2}},
$$

with $m=1,2$, or 3 and $\boldsymbol{\Delta}(\mathbf{k})=\mathbf{I}-c_{0} \hat{\mathbf{G}}^{p}(\mathbf{k})$ being the Green operator for the flux vector, (A13) and (A15) show that

$$
\begin{aligned}
\hat{\mathbf{E}}^{c}\left(\tilde{\mathbf{k}}, x_{3}\right) & =\mathbf{P}\left(\tilde{\mathbf{k}}, x_{3}\right) \mathbf{K}^{-1}(\tilde{\mathbf{k}}) \mathbf{Q}(\tilde{\mathbf{k}}) \\
& =-\mathbf{P}\left(\tilde{\mathbf{k}}, x_{3}\right) \mathbf{K}^{-1}(\tilde{\mathbf{k}}) \sum_{k_{3}} \mathbf{S}(\mathbf{k}) \hat{\mathbf{p}}(\mathbf{k}),
\end{aligned}
$$

where $\tilde{\mathbf{k}} \neq \mathbf{0}$ and $\mathbf{S}(\mathbf{k})$ is a $2 \times 3$ matrix whose components are provided by

$$
S_{1 m}(\mathbf{k})=\Delta_{3 m}(\mathbf{k}) e^{\frac{k_{3} t}{2}}, \quad S_{2 m}(\mathbf{k})=\Delta_{3 m}(\mathbf{k}) e^{-\frac{i_{3} t}{2}} .
$$

By expressing Eq. (27) in the Fourier space such as

$$
\hat{\mathbf{E}}^{c}\left(\tilde{\mathbf{k}}, x_{3}\right)=-\int_{-\frac{f}{2}}^{\frac{f}{2}} \hat{\mathbf{G}}^{c}\left(\tilde{\mathbf{k}}, x_{3}, x_{3}^{\prime}\right) \hat{\mathbf{p}}\left(\tilde{\mathbf{k}}, x_{3}^{\prime}\right) d x_{3}^{\prime},
$$

and accounting for (A20), the Fourier transform of the Green operator $\mathrm{G}^{c}$ is given by

$$
\hat{\mathbf{G}}^{c}\left(\tilde{\mathbf{k}}, x_{3}, x_{3}^{\prime}\right)=\mathbf{P}\left(\tilde{\mathbf{k}}, x_{3}\right) \mathbf{K}^{-1}(\tilde{\mathbf{k}}) \sum_{k_{3}} \mathbf{S}(\mathbf{k}) e^{-i k_{3} x_{3}^{\prime}}, \quad \forall \tilde{\mathbf{k}} \neq \mathbf{0} .
$$

In particular, for $\mathbf{k}=\mathbf{0}$, due to the periodic boundary conditions on $\partial Z_{l}$ and on $\partial Z^{ \pm}$in (24), the Fourier transform of the intensity solution field $\mathbf{E}^{P}(\mathbf{x})$ is therefore equal to zero, i.e., $\hat{\mathbf{E}} P(\mathbf{k}=\mathbf{0})=\mathbf{0}$. This implies immediately that $\hat{\mathbf{G}} \boldsymbol{P}(\mathbf{k}=$ $0)=0$. Similarly, due to the periodic boundary condition on $\partial Z_{l}$ in (26), the Fourier transform components of the intensity solution field $\mathbf{E}^{c}(\mathbf{x})$ at $\tilde{\mathbf{k}}=\mathbf{0}$ are null in-plane, $\hat{E}_{\alpha}^{c}(\tilde{\mathbf{k}}=$ $\left.\mathbf{0}, x_{3}\right)=0$, but non-null out-of-plane, $\hat{E}_{3}^{c}\left(\tilde{\mathbf{k}}=\mathbf{0}, x_{3}\right) \neq 0$. More precisely, by using the boundary condition (A10) and the differential equation that is the second expression in (A7), the non-null out-of-plane component $\hat{E}_{3}^{c}\left(\tilde{\mathbf{k}}=\mathbf{0}, x_{3}\right)$ is given by

$$
\hat{E}_{3}^{c}\left(\tilde{\mathbf{k}}=\mathbf{0}, x_{3}\right)=-\frac{1}{c_{0}} \sum_{k_{3}} \hat{q}_{3}^{P}\left(\tilde{\mathbf{k}}=\mathbf{0}, k_{3}\right) e^{\frac{i_{3} t}{2}} .
$$

It is easy to check from (A6) together with (A18) that $\hat{q}_{3}^{p}(\tilde{\mathbf{k}}=$ $\left.0, k_{3}\right)=0$ for any $k_{3} \neq 0$, and therefore

$$
\hat{E}_{3}^{c}\left(\tilde{\mathbf{k}}=\mathbf{0}, x_{3}\right)=-\frac{1}{c_{0}} \hat{p}_{3}(\mathbf{k}=\mathbf{0}) .
$$

This equation allows us to derive the expression of $\hat{\mathbf{G}}^{c}\left(\tilde{\mathbf{k}}, x_{3}, x_{3}^{\prime}\right)$ at $\tilde{\mathbf{k}}=\mathbf{0}$ as follows:

$$
\hat{\mathbf{G}}^{c}\left(\tilde{\mathbf{k}}=\mathbf{0}, x_{3}, x_{3}^{\prime}\right)=\frac{1}{c_{0}} \mathbf{e}_{3} \otimes \mathbf{e}_{3} .
$$

As expressed previously, the Green operator defined by (28) together with (A18) and (A23) is related to a periodic distribution of sources within the plate. It is used in the paper for computing the Green operator for the infinite plate and nonperiodic polarization by using large enough values of the in-plane period.
[1] Z. Hashin and S. Shtrikman, J. Appl. Phys. 33, 3125 (1962).

[2] M. J. Beran, Statistical Continuum Theories (Wiley, New York, 1968).

[3] N. Phan-Thien and G. W. Milton, Proc. R. Soc. London, Ser. A 380, 333 (1982).

[4] D. C. Pham, Int. J. Eng. Sci. 31, 11 (1993).

[5] D. C. Pham, Acta Mechan. 121, 177 (1997).

[6] D. C. Pham, Mech. Mater. 27, 249 (1998).

[7] S. Torquato, Random Heterogeneous Media (Springer, New York, 2002).

[8] R. Landauer, in Electrical, Transport and Optical Properties of Inhomogeneous Media, edited by J. C. Garland and D. B. Tanner (AIP, New York, 1978).

[9] N. Norris, A. J. Callegari, and P. J. Sheng, J. Mech. Phys. Solids $33,525(1985)$.

[10] N. Phan-Thien and D. C. Pham, Int. J. Eng. Sci. 38, 73 (2000)

[23] T. K. Nguyen, K. Sab, and G. Bonnet, Eur. J. Mech. A/Solids 28, 1051 (2009).

[24] C. P. Wong and R. S. Bollampally, J. Appl. Polym. Sci. 74, 3396 (1999).

[25] T. K. Nguyen, K. Sab, and G. Bonnet, Int. J. Solids Struct. 45, 6518 (2008).

[26] S. Torquato and F. Lado, J. Phys. A 18, 141 (1985).

[27] W. J. Drugan and J. R. Willis, J. Mech. Phys. Solids 44, 497 (1996).
[11] D. C. Pham, Int. J. Heat Mass Transf. 51, 3355 (2008).

[12] T. D. Sermergor, Theory of Elasticity of Micro-inhomogeneous Media (Nauka, Moscow, 1977).

[13] J. J. McCoy, Q. Appl. Math. 37, 137 (1979).

[14] V. Mityushev, Proc. R. Soc. London, Ser. A 455, 2513 (1999).

[15] V. Mityushev, Appl. Math. Opt. 44, 17 (2001)

[16] L. Berlyand and V. Mityushev, J. Stat. Phys. 102, 115 (2001).

[17] W. F. Brown, J. Chem. Phys. 23, 1514 (1955).

[18] A. K. Sen and S. Torquato, Phys. Rev. B 39, 4504 (1989).

[19] D. C. Pham and S. Torquato, J. Appl. Phys. 94. 6591 (2003).

[20] A. G. Kolpakov, J. Mech. Phys. Solids 46, 1039 (1998).

[21] A. G. Kolpakov, J. Mech. Phys. Solids 47, 2075 (1999).

[22] A. G. Kolpakov and I. G. Sheremet, J. Appl. Math. Mech. 63, 633 (1999)

[28] J. R. Willis, in Mechanics of Solids: The R. Hill 60th Anniversary Volume, edited by H. G. Hopkins and M. J. Sewell (Pergamon, Oxford, 1982), p. 653.

[29] W. J. Drugan, J. Mech. Phys. Solids 51, 1745 (2003).

[30] H. L. Weisseberg, J. Appl. Phys. 34, 2636 (1963).

[31] C. Gasquet and P. Witomski, Analyse de Fourier et Applications (Masson, Paris, 2000).

[32] S. Kassbohm, W. H. Muller, and R. Fessler, Comput. Mater. Sci. 32,387 (2005). 